\title{
Modeling Contact Structures in Biology
}

CARLOS CASTILLO-CHAVEZ

332 Warren Hall Biometrics Unit

Cornell University, Ithaca NY 14853-7801

JORGE X. VELASCO-HERNANDEZ

322 Warren Hall Biometrics Unit

Cornell University, Ithaca NY 14853-7801

Departamento El Hombre y su Ambiente

Universidad Autonoma Metropolitana-Xochimilco

Mexico, D.F.

\section{SAMUEL FRIDMAN}

322 Warren Hall Biometrics Unit

Cornell University, Ithaca NY 14853-7801

Abstract. Social dynamics have had a strong impact on the development of theoretical epidemiology over the last six years. Interactions or contacts between individuals have traditionally been modeled using the mass-action law or proportionate mixing, giving limited understanding of effects that the environment-changing social structure-has on disease dynamics. Furthermore, while gender plays a central role in the dynamics of sexually-transmitted diseases, the use of two-sex modeis has been rare. In this article, we review briefly our mixing/pair formation framework and illustrate its application to population models of the type currently used in demography, epidemiology, and social dynamics. A new application to frequency dependent competitive interactions is discussed in more detail. Connections between deterministic and stochastic processes are presented. The results of the simulations of a demographic two-sex stochastic model that follows the dynamics of pairs are presented.

Key words. Epidemiology, social dynamics, demography, food web dynamics, mixing/pair formation, contact rates, consumer-resource interactions, predator-prey systems, differential equation models, vector transmitted diseases, HIV/AIDS, sexually-transmitted diseases, stochastic processes. 


\section{INTRODUCTION.}

The transmission of diseases, genetic characteristics, or cultural traits is influenced by many factors including the contact/social structure of the interacting subpopulation, that is, the social environment. Classical demography (see MacKendrick 1926, Lotka 1922, and Leslie 1945) ignores social dynamics and usually concentrates on the birth and death processes of female populations under the assumption that they have reached a stable age distribution. They usually ignore the specific mating/contact structure of the population. The incorporation of mating structures or marriage functions, as they are commonly referred to in human demography, was pioneered by Kendall (1949) and Keyfitz (1949). However, despite the fact that their work was extended by Parlett (1972), Fredrickson (1971), McFarland (1972), and Pollard (1973) two decades ago, their impact on demography, epidemiology, and population biology has been minimal.

The grim scenario due to the HIV/AIDS epidemic has accelerated the pace at which social dynamics have been incorporated into epidemiological models. Researchers are developing new models and innovative modelling approaches to help us identify and/or improve our understanding of the mechanisms responsible for HIV transmission; for example, there has been intensive research activity on looking at the effects of social dynamics, the immune system variability, etc. in HIV dynamics as well as on the development of methods for the evaluation of competing control measures.

Dietz (1988a) and Dietz and Hadeler (1988) have brought to the forefront-and for the first time in epidemiology-models that incorporate heterogeneity through the processes of pair formation and dissolution. Researchers have shown that epidemic models can be very sensitive to changes in the sexual/social mixing structure of interacting subpopulations as they may not only have different quantitative dynamics but also distinct global dynamics.

The work that we (this generic "we" includes many collaborators who will be cited throughout the text) have conducted over the last few years tas had as a major component the development of a mathematical framework for the systematic incorporation of very general contact structures. The modeling approach outlined in Section 2 of this manuscript has been used to develop models for the study of disease dynamics, the dynamics of frequency-dependent predation in heterogeneously mixing populations (food web dynamics), transmission dynamics of cultural traits, social dynamics, general demographic processes, etc. In addition, we have made serious efforts to connect these models to data (see Rubin et al. 1992, Castillo-Chavez et al. 1992, Hsu Schmitz and Castillo-Chavez 1992) and have participated in the collection of appropriate data (see Crawford et al. 1990).

This manuscript is organized as follows: Section 2 introduces the basic formalism and states the basic theoretical results; Section 3 uses it in the context of demography and social dynamics; Section 4 applies the same approach to model frequency dependent predation and food web dynamics; Section 5 discusses the uses of our approach in the study of models for vector-transmitted diseases. Section 6 illustrates the connections between deterministic and stochastic processes. The results of the simulations of a demographic two-sex stochastic model that follows the dynamics of pairs are presented.

\section{BASIC FRAMEWORK FOR CONTACT STRUCTURES.}

The mass-action law has played a central role in the development of stochastic and deterministic epidemiological models (see Bailey 1975, Anderson 1982, Anderson and May 1991, and references therein). The assumption that the rate of new infections (the incidence) is proportional to the product of susceptibles and infectives in the exposed population has no mathematical significance when one deals with interacting subpopulations that have a constant number of individuals (although it may have an important effect on the interpretation of relevant epidemiological parameters such as the transmission coefficient). However, the mass-action assumption seriously affects the qualitative and quantitative behavior of models with interacting subpopulations of varying size (that is, when the sizes of the interacting subpopulations vary according to deterministic or stochastic rules). Unfortunately, a thorough analysis of basic assumptions such as those implict in the mass-action law was not carried out in a systematic fashion because mathematical epidemiology was growing almost independently of epidemiology (there are some exceptions, e.g., see Hethcote and Yorke 1984).

The HIV/AIDS epidemic revealed the deficiencies and inadequacies of the existing theory. Several questions relevant to the dynamics of heterogeneously mixing populations affected by fatal diseases could not be properly studied under the existing framework. The contact structure of the population must respond at least to the potential population changes due to a heterogeneously 
transmitted fatal disease (or more generally to frequency dependent predation). The importance of the contact process (well recognized by Ross in his work on malaria, 1911) in frequency-dependent systems has motivated the work that we present in the final volume of this series.

Our general approach for modeling contact processes describes who is mixing or pairing with whom. We let $M(a, t)$ denote the density of males of age a who are not in pairs at time $t$, and let $F\left(a^{\prime}, t\right)$ denote the density of females of age $a^{\prime}$ who are not in pairs at time $t$. Pairing is defined through the mixing functions:

and we let

$$
\begin{aligned}
\mathrm{p}\left(\mathrm{a}, \mathrm{a}^{\prime}, t\right)= & \text { proportion of partnerships of males of age a with females } \\
& \text { of age } \mathrm{a}^{\prime} \text { at time } t \\
\mathrm{q}\left(\mathrm{a}, \mathrm{a}^{\prime}, t\right)= & \text { proportion of partnerships of females of age } \mathrm{a}^{\prime} \text { with } \\
& \text { males of age a at time } t,
\end{aligned}
$$

$$
\begin{aligned}
C(a, t)= & \text { expected or average number of partners of a male of } \\
& \text { age a at time } t \text { per unit time, } \\
D\left(a^{\prime}, t\right)= & \begin{array}{l}
\text { expected or average number of partners of a female of } \\
\text { age } a^{\prime} \text { at time } t \text { per unit time. }
\end{array}
\end{aligned}
$$

The following natural conditions characterize these mixing functions:

(i) $\mathrm{p}, \mathrm{q} \geq 0$,

(ii) $\int_{0}^{\infty} \mathrm{p}\left(\mathrm{a}, \mathrm{a}^{\prime}, \mathrm{t}\right) \mathrm{da} \mathrm{a}^{\prime}=\int_{0}^{\infty} \mathrm{q}\left(\mathrm{a}^{\prime}, \mathrm{a}, \mathrm{t}\right) \mathrm{da}=1$,

(iii) $p\left(a, a^{\prime}, t\right) C(a, t) M(a, t)=q\left(a^{\prime}, a, t\right) D\left(a^{\prime}, t\right) F\left(a^{\prime}, t\right)$,

(iv) $C(a, t) M(a, t) D\left(a^{\prime}, t\right) F\left(a^{\prime}, t\right)=0 \Rightarrow p\left(a, a^{\prime}, t\right)=q\left(a^{\prime}, a, t\right)=0$.

Condition (ii) is due to the fact that $p$ and $q$ are probabilities. Condition (iii) simply states that the total rate of pair-formation between males of age a and females of age $a^{\prime}$ equals the total rate of pairformation between females of age $a^{\prime}$ and males of age a (all per unit time and age). Condition (iv) says that there is no mixing in the age and activity levels where there are no active individuals; i.e., on the set $\mathscr{\Psi}(\mathrm{t})=\left\{\left(\mathrm{a}, \mathrm{a}^{\prime}, \mathrm{t}\right): \mathrm{C}(\mathrm{r}, \mathrm{a}, \mathrm{t}) \mathrm{M}(\mathrm{a}, \mathrm{t}) \mathrm{D}\left(\mathrm{a}^{\prime}, \mathrm{t}\right) \mathrm{F}\left(\mathrm{a}^{\prime}, \mathrm{t}\right)=0\right\}$.

The pair $(p, q)$ is called a two-sex mixing function if and only if it satisfies axioms (i-iv). Further, a two-sex mixing function is called separable if and only if

$$
p\left(a, a^{\prime}, t\right)=p_{1}(a, t) p_{2}\left(a^{\prime}, t\right) \text { and } q\left(a, a^{\prime}, t\right)=q_{1}(a, t) q_{2}\left(a^{\prime}, t\right) \text {. }
$$

If we let

$$
h_{p}(a, t)=C(a, t) M(a, t)
$$

and

$$
h_{q}(a, t)=D(a, t) F(a, t)
$$

then, omitting $t$ to simplify the notation, one has the following results (see Busenberg and CastilloChavez 1989, 1991; Castillo-Chavez and Busenberg 1991):

Result 1 The only two-sex separable mixing function satisfying conditions (i-iv) is given by the Ross solution $(\overline{\mathrm{p}}, \overline{\mathrm{q}})$, where

$$
\begin{gathered}
\bar{p}\left(a^{\prime}\right)=\frac{h_{q}\left(a^{\prime}\right)}{\int_{0}^{\infty} h_{p}(u) d u}, \\
\bar{q}(a)=\frac{h_{p}(a)}{\int_{0}^{\infty} h_{q}(u) d u} .
\end{gathered}
$$

We named this solution the Ross solution because Ross (1911) was aware of the importance and necessity of Axiom (c) and used it in his model for malaria, although he only used one vector and one 
host type. This fact was clearly pointed out by Lotka (1923) in his review of Ross's work on malaria models (Ross also outlined the potential use of this work in models for STD's).

Result 2 Any solution of Axioms (i)-(iv) can be written as a multiplicative perturbation of the Ross solution $(\bar{p}, \bar{q})$. The perturbations are parametrized by matrices that measure the affinities/preferences of individuals.

In the next sections we discuss the applications of this framework in a variety of settings. We first outline its use in demographic and social contexts and then provide a new application of this approach in the context of frequency-dependent predation and food web dynamics. Finally, we discuss its use in epidemiology and its implementation in stochastic frameworks (Markov chain models).

\section{DEMOGRAPHIC AND SOCIAL DYNAMIC MODELS.}

Classical demographic models that consider pairs and follow the dynamics of pairs have been studied by Kendall (1949), Keyfitz (1949), Parlett (1972), Fredrickson (1971), McFarland (1972), and Pollard (1973) and have been extended to epidemiology by Dietz and Hadeler (1988), Dietz (1988a), Hadeler (1989a,b), Hadeler and Nagoma (1990), and Waldstätter (1989). Their approach is based on the use of a nonlinear function $\psi$ to model the process (rate) of pair formation. This mixing/pair formation function is assumed to satisfy the Fredrickson/McFarland $(1971,1972)$ properties:

$$
\psi(0, \mathrm{~F})=\psi(\mathrm{M}, 0)=0 .
$$

In the absence of either males or females there will be no heterosexual pair formation. $\psi(\alpha \mathrm{M}, \alpha \mathrm{F})=\alpha \psi(\mathrm{M}, \mathrm{F})$ for all $\alpha, \mathrm{M}, \mathrm{F} \geq 0$.

If the sex ratio remains constant, then the increase in the rate of pair formation is assumed to be proportional to total population size. $\psi(\mathbf{M}+\mathrm{u}, \mathrm{F}+\mathrm{v}) \geq \psi(\mathbf{M}, \mathbf{F})$ for all $\mathrm{u}, \mathrm{v}, \mathrm{F}, \mathrm{M} \geq \mathbf{0}$. Increases in the number of males and/or females does not decrease the rate of pair formation.

Condition (b) implies that all mixing functions are of the form

$$
\psi(\mathrm{M}, \mathrm{F})=\mathrm{M} \mathrm{g}\left(\frac{\mathrm{F}}{\mathrm{M}}\right)=\mathrm{F} \mathrm{h}\left(\frac{\mathrm{M}}{\mathrm{F}}\right),
$$

where $h$ and $g$ are functions of one variable.

Examples of mixing functions satisfying the above axioms include:

and

$$
\begin{aligned}
& \psi(\mathrm{M}, \mathrm{F})=\mathrm{k} \min (\mathrm{M}, \mathrm{F}), \quad \mathrm{k} \text { is a constant } \\
& \psi(\mathrm{M}, \mathrm{F})=\mathrm{k} \sqrt{\mathrm{MF}}
\end{aligned}
$$

$$
\psi(\mathrm{M}, \mathrm{F})=2 \mathrm{k} \frac{\mathrm{MF}}{\mathrm{M}+\mathrm{F}} .
$$

The simplest demographic model that takes into account pair-formation is constructed by balacing the rates of flows between the different compartments/subpopulations; that is, by keeping track of the transition rates associated with the transfer of individuals and pairs of individuals (couples) as they form or dissolve pairings. To state the explicit equations: let $\sigma$ denote the rate of pair dissolution, $\mu$ denote the natural mortality rate, $\Lambda$ denote the "recruitment" rate, and $\mathrm{W}$ denote the number of (heterosexual) pairs. Then Kendall's demographic model is described by the following set of equations:

$$
\begin{aligned}
\frac{\mathrm{dM}}{\mathrm{dt}} & =\Lambda-\mu \mathrm{M}+(\sigma+\mu) \mathrm{W}-\psi(\mathrm{M}, \mathrm{F}) \\
\frac{\mathrm{dF}}{\mathrm{dt}} & =\Lambda-\mu \mathrm{F}+(\sigma+\mu) \mathrm{W}-\psi(\mathrm{M}, \mathrm{F}) \\
\frac{\mathrm{dW}}{\mathrm{dt}} & =-(\sigma+2 \mu) \mathrm{W}+\psi(\mathrm{M}, \mathrm{F}) .
\end{aligned}
$$

If $\Lambda, \mu$, and $\sigma$ are constant, then there is always a globally stationary solution $(M, F, W)$, where $W$ is 
determined by non-trivial solutions to the equation

$$
\psi\left(\frac{\Lambda}{\mu}-\mathrm{W}, \frac{\Lambda}{\mu}+\mathrm{W}\right)=(\sigma+2 \mu) \mathrm{W} .
$$

For references to this and related results see the work of (Dietz and Hadeler 1988, Waldstätter, 1989). Extensions of this model that incorporate the age structure of a population have been carried out by Hadeler $(1989 a, b)$.

We now specify an age-structured demographic model equivalent to those studied by Hadeler $(1989 a, b)$ but using the framework of Section 2. Specifically, let $f\left(a^{\prime}, t\right)$ and $m(a, t)$ denote the agespecific densities for single males and single females respectively, and assume that $\mathrm{C}$ and $\mathrm{D}$ are defined in Section 2, and $\mu_{\mathrm{m}}$ and $\mu_{\mathrm{f}}$ are functions of age (the mortality rates for males and females). In addition, let $W\left(a, a^{\prime}, t\right)$ denote the age-specific density of heterosexual pairs (where a denotes the age of the male and $a^{\prime}$ the age of the female). Then, using the two-sex mixing functions $p$ and $q$ of Section 2, we arrive at the following demographic model for heterosexual populations with pairing:

$$
\begin{aligned}
\frac{\partial \mathrm{m}}{\partial \mathrm{t}}+\frac{\partial \mathrm{m}}{\partial \mathrm{a}}=-\mathrm{C}(\mathrm{a}) \mathrm{m}(\mathrm{a}, \mathrm{t}) & -\mu_{\mathrm{m}}(\mathrm{a}) \mathrm{m}(\mathrm{a}, \mathrm{t})+\int_{0}^{\infty}\left[\mu_{\mathrm{f}}\left(\mathrm{a}^{\prime}\right)+\sigma\right] \mathrm{W}\left(\mathrm{a}, \mathrm{a}^{\prime}, \mathrm{t}\right) \mathrm{d \textrm {a } ^ { \prime }}, \\
\frac{\partial \mathrm{f}}{\partial \mathrm{t}}+\frac{\partial \mathrm{f}}{\partial \mathrm{a}^{\prime}}=-\mathrm{D}\left(\mathrm{a}^{\prime}\right) \mathrm{f}\left(\mathrm{a}^{\prime}, \mathrm{t}\right) & -\mu_{\mathrm{f}}\left(\mathrm{a}^{\prime}\right) \mathrm{f}\left(\mathrm{a}^{\prime}, \mathrm{t}\right)+\int_{0}^{\infty}\left[\mu_{\mathrm{m}}(\mathrm{a})+\sigma\right] \mathrm{W}\left(\mathrm{a}, \mathrm{a}^{\prime}, \mathrm{t}\right) \mathrm{da} \\
\frac{\partial \mathrm{W}}{\partial \mathrm{t}}+\frac{\partial \mathrm{W}}{\partial \mathrm{a}}+\frac{\partial \mathrm{W}}{\partial \mathrm{a}^{\prime}}=\mathrm{D}\left(\mathrm{a}^{\prime}\right) \mathrm{f}\left(\mathrm{a}^{\prime} \mathrm{t}\right) \mathrm{q}\left(\mathrm{a}, \mathrm{a}^{\prime}, \mathrm{t}\right) & \quad-\left[\mu_{\mathrm{f}}\left(\mathrm{a}^{\prime}\right)+\mu_{\mathrm{m}}(\mathrm{a})+\sigma\right] \mathrm{W}\left(\mathrm{a}, \mathrm{a}^{\prime}, \mathrm{t}\right) .
\end{aligned}
$$

To complete this model we must specify the initial and boundary conditions. To this effect we let $\lambda_{m}$ and $\lambda_{f}$ denote the female age-specific fertility rates, and let $m_{0}, f_{0}$, and $w_{0}$ denote the intial age densities. Hence, the initial and boundary conditions are given by

$$
\begin{aligned}
\mathrm{m}(0, \mathrm{t}) & =\int_{0}^{\infty} \lambda_{\mathrm{m}}\left(\mathrm{a}^{\prime}\right) \mathrm{N}_{\mathrm{f}}\left(\mathrm{a}^{\prime}, \mathrm{t}\right) \mathrm{da^{ \prime }}, \\
\mathrm{f}(0, \mathrm{t}) & =\int_{0}^{\infty} \lambda_{\mathrm{f}}\left(\mathrm{a}^{\prime}\right) \mathrm{N}_{\mathrm{f}}\left(\mathrm{a}^{\prime}, \mathrm{t}\right) \mathrm{da^{ \prime }}, \\
\mathrm{W}(0,0, \mathrm{t}) & =0 \\
\mathrm{f}(\mathrm{a}, 0) & =\mathrm{f}_{\mathrm{o}}(\mathrm{a}), \mathrm{m}(\mathrm{a}, 0)=\mathrm{m}_{\mathrm{o}}(\mathrm{a}), \mathrm{W}\left(\mathrm{a}, \mathrm{a}^{\prime}, 0\right)=\mathrm{W}_{\mathrm{o}}\left(\mathrm{a}, \mathrm{a}^{\prime}\right)
\end{aligned}
$$

where

$$
N_{f}\left(a^{\prime}, t\right)=\int_{0}^{\infty} W\left(a, a^{\prime}, t\right) d a .
$$

We observe that $N_{f}$ and $f+N_{f}$ satisfy the following set of equations:

$$
\left(\frac{\partial}{\partial \mathrm{t}}+\frac{\partial}{\partial \mathrm{a}^{\prime}}\right)\left(\mathrm{f}+\mathrm{N}_{\mathrm{f}}\right)=-\mu_{\mathrm{f}}\left(\mathrm{a}^{\prime}\right)\left[\mathrm{f}+\mathrm{N}_{\mathrm{f}}\right]
$$

and

$$
\begin{aligned}
\left(\frac{\partial}{\partial \mathrm{t}}+\frac{\partial}{\partial \mathrm{a}^{\prime}}\right) \mathrm{N}_{\mathrm{f}}=\mathrm{D}\left(\mathrm{a}^{\prime}\right) \mathrm{f}\left(\mathrm{a}^{\prime}, \mathrm{t}\right) & \\
- & {\left[\mu_{\mathrm{f}}\left(\mathrm{a}^{\prime}\right)+\sigma\right] \mathrm{N}_{\mathrm{f}} } \\
& -\int_{0}^{\infty} \mu_{\mathrm{m}}(\mathrm{a}) \mathrm{W}\left(\mathrm{a}, \mathrm{a}^{\prime}, \mathrm{t}\right) \mathrm{d} \mathrm{a} .
\end{aligned}
$$


If we let $\sigma \rightarrow \infty$ (while fixing a, $t$, and $\mu_{m}(a)$ constant) then $\mathrm{N}_{f}(\mathrm{a}, \mathrm{t}, \mathrm{t}) \rightarrow 0^{+}$and formally Equation (9) approaches the classical MacKendrick/Von Foerster model. However, since in the model given by Equations (7)-(9) only pairs reproduce we can not recover the classical boundary condition (this situation is easily corrected if one uses Dietz's definition of a pair, 1988a). For some preliminary analysis of this model see Castillo-Chavez et al. (1991).

Models like (7) are useful because of the importance of the mating system and the average duration of partnerships in the transmission dynamics of cultural traits. Superficially, the cultural transmission of traits appears similar to genetic transmission. However, there are further complications, as the inheritance of social traits such as language and religion is influenced by the level of heterogeneity of the population at large. Previous approaches to the modelling of cultural trait transmission have been either very specific, as in the bilingual competition model of Baggs and Freedman (1990), or quite general, albeit within very restricted or rigid frameworks. Cavalli-Sforza and Feldman (1981) and Boyd and Richerson (1985) assume that cultural transmission happens once per generation, e.g., at birth. Hence they exclude many situations including religious conversion while at the same time their model is riddled with the usual inherent limitations of models with nonoverlapping generations. In Lubkin et al. (1992), we have developed a flexible framework to study the transmission dynamics of cultural traits in age-structured populations with overlapping generations. The flexibility comes from the incorporation of pairings, partnership duration, and "arbitrary" mating systems. In Lubkin et al. (1992) examples are provided that include the melting pot, biparental determination, and maternal determination models. Finally, we note that the models of the type here constructed do not represent just an exercise in modelling, as their use has begun to have a serious impact in the area of sociology, epidemiology, immunology, and ecology. The current revisions of the theory have increased the number of interactions between a large number of scientists from biology, sociology, epidemiology, statistics. and mathematics. The large number of interdisciplinary conferences and workshops that have brought these groups together over the last five years has radically changed the fields of mathematical population dynamics and mathematical epidemiology.

\section{FREQUENCY DEPENDENT PREDATION MODELS.}

Here we shift gears and discuss applications of the framework of Section 2 to situations in which frequency-dependent predation is important. Our emphasis is on the description of prey selection, competition for common resources (see Pimm, 1982, 1988), interaction strength (Pimm and Kitching, 1988), and their relation to food web dynamics. In this section, we define a food web as a network composed of biological species interacting through frequency-dependent predation and competition and illustrate our approach with the use of a simple three-level food web ( top predators, intermediate species, bottom species).

The mechanisms by which predators select prey for their diet has been the subject of intensive research (Akre et al., 1979; Cock, 1978; Levin and Segel, 1982; Chesson, 1978, 1983; Gendron, 1987; Oaten and Murdoch, 1975; Teramoto et al., 1979). A given predator's diet is, in principle, not necessarily related to the abundance of the different prey types available (Gendron, 1987). From a phenomenological point of view, this outcome can be seen as the product of a density-dependent risk of being captured and the density of other alternative prey (Gendron, 1987). To model these interactions we let $c_{i j}$ denote the average per capita number of effective contacts (leading to a successful meal) between predators of type $i$ and prey of type $j$ per unit time; while $r_{j i}$ denotes the average per capita number of prey of type $j$ captured by predators of type $i$ per unit time. If $T_{i}(t)$ denotes the number or density of predators of type $i$ at time $t$ and $N_{j}(t)$ denotes the number or density of prey of type $j$ at time $t$, we must then have that

$$
\mathrm{T}_{i} \mathrm{c}_{i j}=\mathrm{N}_{j} \mathrm{r}_{j i} \text {. }
$$

By setting

one has

$$
\begin{gathered}
\mathrm{c}_{i}:=\sum_{j}^{m} \mathrm{c}_{i j}, \quad \mathrm{r}_{j}:=\sum_{i}^{n} \mathrm{r}_{j i}, \\
\mathrm{c}_{i} \mathrm{~T}_{i} \mathrm{c}_{i j} \mathrm{c}_{i}^{-1}=\mathrm{r}_{j} \mathrm{~N}_{j} \mathrm{r}_{j i} \mathrm{r}_{j}^{-1} . \\
\mathrm{p}_{i j}=\mathrm{c}_{i j} \mathrm{c}_{i}^{-1} \text { and } \mathrm{q}_{j i}=\mathrm{r}_{j i} \mathrm{r}_{j}^{-1},
\end{gathered}
$$

Defining

permits the interpretation of $\mathrm{p}_{i j}$ as the proportion of prey of type $\mathrm{j}$ on the diet of the $i$ th predator, and $\mathrm{q}_{j i}$ as the proportion of the $\mathrm{j}$ th prey type consumed by the $\mathrm{i}$ th predator. Hence the matrix 
$\left(\mathrm{p}_{i k}, \mathrm{q}_{k i}\right)$ satisfies the discrete analog of properties (i)-(iv). Using these definitions we introduce the concept of a mixing matrix in the context of a finite number of interacting subpopulations:

Definition 1: The matrix $\left(\mathrm{p}_{i k}, \mathrm{q}_{k i}\right)$ is called a mixing/contact matrix if and only if it satisfies the following properties:

(di) $0 \leq \mathrm{p}_{i k} \leq 1$, and $0 \leq \mathrm{q}_{k i} \leq 1$,

(dii) $\sum_{k=1}^{m} \mathrm{p}_{i k}=1=\sum_{i=1}^{n} \mathrm{q}_{k i}$,

(diii) $c_{i} \mathrm{~T}_{i} \mathrm{p}_{i k}=\mathrm{r}_{k} \mathrm{~N}_{k} \mathrm{q}_{k i}$, for $\mathrm{i}=1, \cdots, \mathrm{n}$ and $\mathrm{j}=1, \cdots, \mathrm{m}$.

(div) If for some $i, 1 \leq i \leq n$ and/or some $j, 1 \leq j \leq m$ we have that $c_{i} r_{k} T_{i} N_{k}=0$, then we define $\mathrm{p}_{i k} \equiv \mathrm{q}_{k i} \equiv 0$.

Condition (diii) is interpreted as a conservation of contacts law or group reversibility property. The total number of contacts per unit time of predators of type $i$ and prey of type $j$ has to be equal to the number of contacts between prey of type $j$ with predators of type $i$. The condition relates the rates at which k-prey are captured by i-predators $c_{i} p_{i k}$ and $r_{k} q_{k i}$. With k-prey and i-predator abundance $N_{k}$ and $T_{k}$, we are introducing dynamic concepts involving biological species interactions. Condition (div) asserts that the mixing of nonexistent subpopulations, either of prey or predators, cannot be arbitrarily defined. The symmetry involved in the total number of contacts required for predatorprey, consumer-resource or host-parasite interactions is an obvious fact that has not been fully explored until very recently (see Castillo-Chavez and Busenberg, 1991).

We re-derive expressions for the mixing probabilities that allow for the incorporation of handling times. Let $\tau_{i}$ be the total relative time (e.g., the average total time used by predator species i divided by the smallest average handling predator time or a similar weighted average) spent foraging by an average predator of species $i$, and let $\sigma_{i j}$ denote the per capita average handling time spent by a predator of species $i$ on prey in group $j, i=1, \ldots, n, j=1, \ldots, m$. Then

$$
\tilde{\sigma}_{i}=\sum_{k=1}^{m} \sigma_{i k} c_{i k}
$$

denotes the mean per capita handling time of predators of group $i$. The searching time (the available time that predators of species $i$ have to find prey suitable for consumption) of predators of type $i$ is $\tau_{i}-\tilde{\sigma}_{i^{\circ}}$ Hence, the number of contacts that an average predator of type $i$ has with prey in group $j$ during the searching time is

$$
\mathrm{c}_{i j}=\left(\tau_{i}-\tilde{\sigma}_{i}\right) \mathrm{b}_{i j} \mathrm{~N}_{j},
$$

where $b_{i j}$ denotes the proportion of contacts that result in a capture of prey from group $j$ by predator species $i$.

$$
\text { Substituting (10) in (11) gives } \tilde{\sigma}_{i}=\left(\tau_{i}-\tilde{\sigma}_{i}\right) \sum_{k=1}^{\mathrm{m}} \sigma_{i k} \mathrm{~b}_{i k} \mathrm{~N}_{k}
$$

Solving for $\tilde{\sigma}_{i}$ leads to

$$
\tilde{\sigma}_{i}=\frac{\tau_{i} \sum_{k=1}^{\mathrm{m}} \sigma_{i k} \mathrm{~b}_{i k} \mathrm{~N}_{k}}{1+\sum_{k=1}^{\mathrm{m}} \sigma_{i k} \mathrm{~b}_{i k} \mathrm{~N}_{k}}
$$

while substitution of (12) into (11) leads, after some algebra, to

$$
c_{i j}=\frac{\tau_{i} b_{i j} N_{j}}{1+\sum_{k=1}^{m} \sigma_{i k} b_{i k} N_{k}} .
$$

From the ratio $c_{i j} / c_{i}$, we conclude that the probability of an effective contact between a predator of species $i$ and prey of group $j$ is

$$
\mathrm{p}_{i j}=\frac{\mathrm{b}_{i j} \mathrm{~N}_{j}}{\sum_{\mathrm{k}=1}^{\mathrm{m}} \mathrm{b}_{i \mathrm{k}} \mathrm{N}_{\mathrm{k}}} .
$$

To derive the probability $q_{j i}$ of an effective contact of predators of group $i$ with prey of species $j$ we solve the relation $\mathrm{T}_{i} \mathrm{c}_{i j}=\mathrm{N}_{j} \mathrm{r} j i$ for $\mathrm{r}_{j i}$. The formula $\mathrm{q}_{j i}=\mathrm{r}_{j i} / \mathrm{r}_{j}$ leads to

$$
\mathrm{q}_{j i}=\frac{\tau_{i} \mathrm{~b}_{i j} \mathrm{~T}_{j}}{1+\sum_{k=1}^{\mathrm{m}} \sigma_{i k} \mathrm{~b}_{i k} \mathrm{~N}_{k}} / \sum_{\mathrm{u}=1}^{\mathrm{n}} \frac{\tau_{\mathrm{u}} \mathrm{b}_{\mathrm{u} j} \mathrm{~T}_{j}}{1+\sum_{k=1}^{\mathrm{m}} \sigma_{\mathrm{u} k} \mathrm{~b}_{\mathrm{u} k} \mathrm{~N}_{k}} .
$$

Equations (13) and (14) satisfy the mixing axioms (di) - (diii). Formula (14) may be interpreted in the following way: $b_{i j}$ is the maximum capt re proportion in the absence of frequency-dependent effects; $\tau_{i} b_{i j} \mathrm{~T}_{i}$ is the number of captures of prey of type $\mathrm{j}$ by predators of type $\mathrm{i}$ during the total foraging time characteristic of the predator species. The numerator of (14) gives the proportion of 
captures of all potential prey of predators of type $i$, while its denominator represents the total number of captures made by all types of predators per unit time; $p_{i j}$ depends only on the weighted relative proportion of prey types, while $q_{j i}$ depends also on the handling times of each predator species involved.

Definition 2 A predator-prey mixing probability is called separable if and only if

$$
\mathrm{p}_{i j}=\mathrm{p}_{i} \tilde{\mathrm{p}}_{j} \quad \text { and } \quad \mathrm{q}_{j i}=\mathrm{q}_{j} \tilde{\mathrm{q}}_{i} .
$$

'To obtain separable solutions from formulae (12) and (13), one requires $b_{i j}=b$ for all indices (i.e., the maximum capture proportion is the same for all predators regardless of the prey type they capture). This assumption leads to the following set of contact probabilities (Ross solutions):

$$
\tilde{\mathbf{p}}_{j}=\frac{\mathrm{N}_{j}}{\sum_{\mathrm{u}=1}^{\mathrm{m}} \mathrm{N}_{\mathbf{u}}},
$$

and

$$
\tilde{\mathbf{q}}_{i}=\frac{\tau_{i} \mathrm{~T}_{i}}{1+\mathrm{b} \sum_{k=1}^{\mathrm{m}} \sigma_{i k} \mathrm{~N}_{k}} / \sum_{\mathrm{k}=1}^{\mathrm{n}} \frac{\tau_{k} \mathrm{~T}_{k}}{1+\mathrm{b} \sum_{\mathrm{u}=1}^{\mathrm{m}} \sigma_{\mathrm{ku}} \mathrm{N}_{\mathrm{u}}} .
$$

Thus, the frequency of a prey type in the diet of a predator depends on the proportion of prey types available, while the presence of a given prey type in the diet of a predator depends on the relative foraging time invested in capturing it. This last factor is commonly associated with the functional response of the predator (see, e.g. Price, 1990).

4.a. The components of predation risk. Gendron (1987) has shown that the components of risk (how likely it is for a prey of any given type to be captured by a predator) can be understood in terms of the following factors:

- The efficiency of the search path.

- The area searched by the predator per unit time.

- The conditional probability of detecting prey.

- The conditional probability of attacking and then capturing detected prey.

Models for predator switching behavior are defined in terms of the frequency of each prey type in the diet of the predator. Specifically, $F_{i}$, the frequency of prey type $i$ is defined as

$$
\mathrm{F}_{i}=\frac{\beta_{i} \mathrm{~N}_{i}}{\sum_{j=1}^{m} \beta_{j} \mathrm{~N}_{j}}
$$

where $\beta_{i}$ is a measure of the relative risk of prey i. Usually $\beta_{\iota}$ is computed by the formula

$$
\beta_{i}=\frac{\mathrm{r}_{i}}{\sum_{j=1}^{m} \mathrm{r}_{j}}
$$

where $r_{i}$ denotes the risk index of species $i$. Frequency-dependent predation requires risk indices which are functions of the relative density of the prey species and give rise to the switching behavior of predators. Generalizations of this switching behavior model useful for statistical analyses are of the form

$$
F_{i}=\frac{f\left(X_{i}\right)}{\sum_{j} f\left(X_{j}\right)}
$$

where $f$ is a nonlinear (usually a polynomial) function of $X_{j}$, the density of prey species $j$ (e.g., Gendron, 1987):

To account for several predator species competing for a collection of prey species, we reformulate equation (17) in the following way

$$
\mathrm{F}_{i j}=\frac{\beta_{i j} \mathrm{~N}_{i}}{\sum_{\mathrm{k}=1}^{m} \beta_{i \mathrm{k}} \mathrm{N}_{\mathrm{k}}} .
$$

The model is complete after the postulation of appropriate functional forms for the relative risks of predation $\beta_{i j}$. These functional forms usually weigh each prey type according to the risk of being captured (see Gendron, 1987). The connection with the mixing theory described before is made by applying our matrix $\left(\mathrm{p}_{i j}, \mathrm{q}_{j \mathrm{i}}\right)$ in (13)-(14) to model $\mathrm{F}_{i j}$ above.

Predation in nature is a selective process and has also been explored in the context of food we' 3 (Pimm, 1982, 1988; Fretwell, 1987). This frequency-dependent process may be d $\lrcorner$ exclusively to frequency-dependent effects--the most numerous prey provides a greater share of the diet of alıy 
predator--or by an active process of preferential prey selection which may be more suitable for the survival of a predator. The formalism introduced by the mixing probabilities describes both processes. Pimm $(1982,1988)$ observes rare as well as common species of prey in predators' diets, thus imposing a ranking in prey species selectivity. This ranking depends on each predator species but it is not transposable to communities. The modeling approach introduced here allows for the incorporation of these effects. In the next sub-section we incorporate the mixing formalism in dynamic models of predator-prey interactions. These models form the basis of our approach to modeling food web dynamics.

4.b. Predator prey interactions and food webs. The general model of predator-prey interaction is given by the system:

$$
N^{\prime}(t)=g[N(t)]-R(N, T) ; \quad T^{\prime}(t)=T(t) G(N, T)-d T(t),
$$

where $G(N, T)$ is the numerical response of the predator, $R(N, T) / T$ is the number of prey consumed relative to prey density or functional response of the predator, and the symbol ' denotes derivative with respect to time. The term $g(N)$ models the growth process of a prey population when predators are absent and $d$ is the density-independent mortality rate of the predator.

A generalized form of the predator-prey model (19) that allows for heterogeneity in prey and predator interactions is given by the following set of equations:

$$
\begin{aligned}
& N_{j}^{\prime}(t)=g_{j}[N(t)]-r_{j} N_{j} \sum_{k=1}^{n} q_{j k} \frac{T_{k}}{T}, \\
& T_{i}^{\prime}(t)=\alpha_{i} T_{i}(t) \sum_{k=1}^{m} r_{k} q_{k i} \frac{N_{k}}{T}-d_{i} T_{i} .
\end{aligned}
$$

Model (20) may also be used to describe the competitive interaction between species that share a spectrum of biotic resources distributed among themselves according to the mixing matrix $\left(\mathrm{p}_{i j}, \mathbf{q}_{j i}\right)$. The first equation in (20) describes the $j t h$ - prey population growing according to $g_{j}[N(t)]$ in the absence of predators.

The term

$$
\mathbf{r}_{\mathbf{j}} \sum_{\mathbf{i}=1}^{\mathrm{n}} \mathrm{q}_{\mathbf{j} i} \frac{\mathrm{T}_{\mathbf{i}}}{\mathrm{T}}
$$

represents the total consumption rate of $\mathrm{N}_{\mathrm{j}}$ by all predators in the community. To obtain the recruitment rate of the predator population we proceed as follows:

The term

$$
\mathrm{r}_{\mathrm{j}} \mathrm{N}_{\mathrm{j}} \mathrm{q}_{\mathrm{ji}} \frac{\mathrm{T}_{\mathrm{i}}}{\mathrm{T}}
$$

represents the total consumption rate of prey $\mathrm{N}_{\mathrm{i}}$ by predators of type $\mathrm{i}$. However, these predators eat not only prey of type $j$ but of all types. Hence to obtain the overall capture rate by type $i$ predators, we have to sum their effect over all prey types obtaining

$$
\mathrm{T}_{i}(\mathrm{t}) \sum_{j=1}^{\mathrm{m}} \mathrm{r}_{\mathrm{j}} \mathrm{q}_{\mathrm{kj}} \frac{\mathrm{N}_{j}}{\mathrm{~T}} \text { : }
$$

The factor $\alpha_{i}$ represents the efficiency of a predator of type $i$ in converting captured prey into predator biomass (offspring). By virtue of the relations

$$
\begin{aligned}
& \mathrm{c}_{i} \mathrm{~T}_{i} \mathrm{p}_{i k}=\mathrm{r}_{k} \mathrm{~N}_{k} \mathrm{q}_{k i}, \\
& \sum_{i=1}^{n} \mathrm{c}_{i} \mathrm{~T}_{i}=\sum_{j=1}^{m} \mathrm{r}_{j} \mathrm{~N}_{j},
\end{aligned}
$$

we have that the number of prey captured equals the number of prey consumed.

Remark 1 In fact we note the following property:

$$
\mathrm{T}_{\mathrm{k}} \sum_{j=1}^{\mathrm{m}} \mathrm{r}_{\mathrm{j}} \mathrm{q}_{\mathrm{jk}} \frac{\mathrm{N}_{j}}{\mathrm{~T}}=\frac{\mathrm{T}_{\mathrm{k}}}{\mathrm{T}} \sum_{j=1}^{\mathrm{m}} \mathrm{r}_{\mathrm{j}} \mathrm{q}_{\mathrm{jk}} \mathrm{N}_{j}=\frac{\mathrm{T}_{\mathrm{k}}}{\mathrm{T}} \sum_{j=1}^{\mathrm{m}} \mathrm{c}_{\mathrm{k}} \mathrm{p}_{\mathrm{kj}} \mathrm{T}_{\mathrm{k}}=\mathrm{c}_{\mathrm{k}} \frac{\mathrm{T}_{\mathrm{k}}}{\mathrm{T}} \mathrm{T}_{\mathrm{k}}
$$

Our simplest food web model considers only three trophic levels (basal, intermediate and top) each with $\ell, m$ and $\mathrm{n}$ species, respectively. The dynamics are specified by the following transfer food web diagram: 


$$
\mathrm{N} \rightarrow \mathrm{Y} \rightarrow \mathrm{T},
$$

where $\mathrm{T}=\left\{\mathrm{T}_{i}\right\}_{1}^{n}$ denotes the top species, $\mathrm{Y}=\left\{\mathrm{Y}_{k}\right\}_{1}^{m}$ the intermediate species and $\mathrm{N}=\left\{\mathrm{N}_{j}\right\}_{1}^{\ell}$ the basal species. The model equations are

$$
\begin{aligned}
& \mathrm{N}_{k}^{\prime}(\mathrm{t})=\mathrm{g}_{k}\left[\mathrm{~N}_{k}(\mathrm{t})\right]-\mathrm{s}_{k} \mathrm{~N}_{k} \sum_{j=1}^{m} \theta_{k j} \frac{\mathrm{Y}_{\mathrm{j}}}{\mathrm{Y}}, \\
& \mathrm{Y}_{j}^{\prime}(\mathrm{t})=\alpha_{\mathrm{j}} \mathrm{Y}_{\mathrm{j}}(\mathrm{t}) \sum_{j=1}^{l} \mathrm{~s}_{k} \theta_{\mathrm{kj}} \frac{\mathrm{N}_{\mathrm{k}}}{\mathrm{Y}}-\mathrm{r}_{\mathrm{j}} \mathrm{Y}_{\mathrm{j}} \sum_{i=1}^{n} \mathrm{q}_{j \mathrm{i}} \frac{\mathrm{T}_{\mathrm{i}}}{\mathrm{T}} \delta_{j} Y_{j},
\end{aligned}
$$

and

$$
\mathrm{T}_{i}^{\prime}(\mathrm{t})=\beta_{\mathrm{i}} \mathrm{T}_{i}(\mathrm{t}) \sum_{j=1}^{m} \mathrm{r}_{\mathrm{j}} \frac{\mathrm{Y}_{j}}{\mathrm{~T}} \mathrm{q}_{j i}-\mathrm{d}_{i} \mathrm{~T}_{i},
$$

where $\alpha_{j}$ and $\beta_{i}$ are coefficients that measure the efficiency of conversion of captured prey into predator biomass, for $j=1, \cdots, m$ and $i=1, \cdots, n$. Also $g_{k}$ denotes the growth law of the kth basal species in the absence of predators.

This model implicitly assumes that all species in level $T$ are linked to all species in level $Y$, and that all species in level $Y$ are linked to all basal species in $N$. There are $(\ell+n) m$ links in this completely connected food web. The contact probabilities $\left(p_{i j}, q_{j i}\right)$ (for encounters between $T$ and $Y$ populations) and $\left(\pi_{i j}, \theta_{j i}\right)$ (for encounters between $Y$ and $N$ populations) satisfy the axioms in Definition 1 as well as the conditions

$$
\begin{aligned}
c_{i} \mathrm{~T}_{i} \mathrm{p}_{i k} & =\mathrm{r}_{k} \mathrm{Y}_{k} \mathrm{q}_{k i}, \\
\mathrm{a}_{k} \mathrm{Y}_{k} \boldsymbol{\pi}_{k j} & =\mathrm{s}_{j} \mathrm{~N}_{j} \boldsymbol{\theta}_{j k},
\end{aligned}
$$

for $\mathrm{i}=1, \cdots, \mathrm{n}, \mathrm{k}=1, \cdots, \mathrm{m}$ and $\mathrm{j}=1, \cdots, \ell$.

We now replace $g_{k}$ by $g_{k}(x)=\lambda_{k}-\mu_{k} \mathrm{x}$ where $\lambda_{k}$ and $\mu_{k}$ are constants representing the recruitment rate and the mortality of the kth population of prey respectively. We obtain

$$
\begin{aligned}
& N_{k}^{\prime}(t)=\lambda_{k}-\mu_{k} N_{k}-s_{k} N_{k} \sum_{j=1}^{m} \theta_{k j} \frac{Y_{j}}{Y}, \\
& Y_{j}^{\prime}(t)=\alpha_{j} Y_{j}(t) \sum_{k=1}^{l} s_{k} \theta_{k j} \frac{N_{k}}{Y}-r_{j} Y_{j} \sum_{i=1}^{n} \frac{T_{i}}{T} q_{j i}-\delta_{j} Y_{j},
\end{aligned}
$$

and

where $\mathrm{N}=\sum_{k=1}^{\ell} \mathrm{N}_{k}$ and $\mathrm{Y}=\sum_{j=1}^{m} \mathrm{Y}_{j}$.

$$
\mathrm{T}_{i}^{\prime}(\mathrm{t})=\beta_{\mathrm{i}} \mathrm{T}_{i}(\mathrm{t}) \sum_{j=1}^{m} \mathrm{r}_{\mathrm{j}} \frac{\mathrm{Y}_{j}}{\mathrm{~T}} \mathrm{q}_{j i}-\mathrm{d}_{i} \mathrm{~T}_{i},
$$

The initial conditions have to satisfy the constraints

$$
\sum_{i=1}^{n} c_{i} \mathrm{~T}_{i}=\sum_{j=1}^{m} \mathrm{r}_{j} \mathrm{Y}_{j} \quad \text { and } \quad \sum_{i=1}^{m} \mathrm{a}_{i} \mathrm{Y}_{i}=\sum_{j=1}^{\ell} \mathrm{s}_{j} \mathrm{~N}_{j}
$$

at time $t=0$. The mixing probabilities in (22a) are assumed to have the simplest possible form; i.e., they will be assumed to be Ross solutions (separable solutions) describing proportionate mixing of captures. Thus we have $p_{i j}=\tilde{p}_{j}, q_{j i}=\tilde{q}_{i}, \pi_{i j}=\tilde{\pi}_{j}$ and $\theta_{j i}=\tilde{\theta}_{i}$

where

$$
\begin{aligned}
& \tilde{\mathrm{p}}_{j}=\left(\frac{\mathrm{Y}_{j}}{\sum_{\mathrm{k}=1}^{m} \mathrm{Y}_{\mathrm{k}}}\right) \\
& \tilde{\mathrm{q}}_{i}=\left[\frac{\rho_{i} \mathrm{~T}_{i}}{1+\mathrm{b} \sum_{k=1}^{m} \sigma_{i k} \mathrm{Y}_{k}} / \sum_{\mathrm{u}=1}^{m} \frac{\rho_{\mathrm{u}} \mathrm{T}_{\mathrm{u}}}{1+\mathrm{b} \sum_{k=1}^{m} \sigma_{\mathrm{u} k} \mathrm{Y}_{k}}\right]
\end{aligned}
$$

and

$$
\begin{aligned}
& \tilde{\pi}_{j}=\left(\frac{\mathrm{N}_{j}}{\sum_{\mathrm{k}=1}^{\ell} \mathrm{N}_{\mathrm{k}}}\right) \\
& \tilde{\theta}_{i}=\left[\frac{\eta_{i} \mathrm{Y}_{i}}{1+\mathrm{e} \sum_{k=1}^{\ell} \omega_{i k} \mathrm{~N}_{k}} / \sum_{\mathrm{u}=1}^{\ell} \frac{\eta_{\mathrm{u}} \mathrm{Y}_{\mathrm{u}}}{1+\mathrm{e} \sum_{k=1}^{\ell} \omega_{\mathrm{u} k} \mathrm{~N}_{k}}\right] .
\end{aligned}
$$

In model (22) we consider the case where the capture of prey by corresponding predators is given by Ross solutions (15) in both the top and the intermediate levels of the food chain. The capture of 
prey is essentially a random process where predators do not show preference for prey of any type. Furthermore, we have that the total populations in each trophic level follow the dynamics specified by the system

$$
\begin{aligned}
& \mathrm{N}^{\prime}(\mathrm{t})=\sum_{k=1}^{\ell}\left(\lambda_{k}-\mu_{\mathrm{k}} \mathrm{N}_{k}\right)-\sum_{\mathrm{k}=1}^{l} \mathrm{~s}_{\mathrm{k}} \mathrm{N}_{\mathrm{k}} \sum_{j=1}^{m} \tilde{\theta}_{\mathrm{j}} \frac{\mathrm{Y}_{\mathrm{j}}}{\mathrm{Y}}, \\
& \mathrm{Y}^{\prime}(\mathrm{t})=\left(\sum_{j=1}^{m} \alpha_{\mathrm{j}} \tilde{\theta}_{\mathrm{j}} \mathrm{Y}_{j}\right)\left(\sum_{k=1}^{\ell} \mathrm{s}_{k} \frac{\mathrm{N}_{k}}{\mathrm{Y}}\right)-\left(\sum_{j=1}^{m} \mathrm{r}_{\mathrm{j}} \mathrm{Y}_{j}\right)\left(\sum_{k=1}^{\mathrm{n}} \tilde{\mathrm{q}}_{k} \frac{\mathrm{T}_{k}}{\mathrm{~T}}\right)-\sum_{j=1}^{m} \delta_{j} \mathrm{Y}_{j}, \\
& \mathrm{~T}^{\prime}(\mathrm{t})=\left(\sum_{i=1}^{n} \beta_{\mathrm{i}} \tilde{\mathrm{q}}_{\mathrm{i}} \mathrm{T}_{i}\right)\left(\sum_{j=1}^{m} \mathrm{r}_{j} \frac{\mathrm{Y}_{j}}{\mathrm{~T}}\right)-\sum_{i=1}^{n} \mathrm{~d}_{i} \mathrm{~T}_{i} .
\end{aligned}
$$

This system is a representation of the dynamic behavior of the trophic species $N, Y$, and $T$ or, in other words, it represents a model for the dynamics of the trophic web as such. Only in very special cases, as it will be shown below, can the RHS of these equations be written in terms of $N, Y$, and $T$, and hence be solved independently of the biological species that constitute each trophic level. Statistical models of food webs deal at this level of organization, therefore incorporating a particular aggregation structure.

The RHS of the equation for either type of predator in the aggregated model (top $T$, and intermediate $\mathrm{Y}$ ) include two of the components of predation. The second factor in the first term describes the availability of prey - which depends on the ratio of prey numbers to predator numbers very much as the expression for the vectorial capacity in vector-transmitted diseases - , and the first factor represents the probability of capturing the encountered prey. The explicit introduction of relaive handling times and the appearance of the ratio of prey to predator density in the predator capture rates indicate its dependence on the number of available prey for each predator type and the behavioral constraints of the predator in the capture and consumption of prey.

The constant equilibrium solutions of model (22) can be found by equating the RHS of each equation to zero. In the simplest case, the mixing probabilities are Ross solutions that involve constant and identical handling or searching times for all types of predator. In this case $\tilde{\theta}_{j}=Y_{j} / Y$ and $\tilde{\mathbf{q}}_{\mathbf{j}}=\mathrm{T}_{\mathbf{i}} / \mathrm{T}$.

Using Remark 1 together with the definitions of the mixing probabilities for the matrices $\left(\tilde{\mathbf{p}}_{\mathbf{j}}\right.$, $\left.\tilde{q}_{\mathbf{i}}\right),\left(\tilde{\pi}_{\mathbf{j}}, \tilde{\theta}_{\mathbf{i}}\right)$, we find the equilibrium values

and

$$
\begin{aligned}
& y_{j}=\frac{r_{j} \sum_{i=1}^{n} \beta_{i} t_{i}^{2}+\delta_{j}}{\alpha_{j} a_{j}}, \text { or } Y_{j}=0, \text { for some } j, j=1, . ., m ; \\
& t_{i}=\frac{d_{i}}{\beta_{i} c_{i}} \text { or } T_{i}=0, \text { for some } i, i=1, \ldots, n ; \\
& N_{k}=\frac{\lambda_{k}}{s_{k} \sum_{i=1}^{m} y_{j}^{2}+\mu_{k}}
\end{aligned}
$$

where $y_{j}=Y_{j} / Y$ and $t_{i}=T_{i} / T$. Notice that with (23) one can determine the equilibrium value of $Y$ from the second equation in (22a), and then by using it and the third equation in (22a) we can find the equilibrium value of $T$. If all species have zero densities except those in the basal web level, one more equilibrium occurs when

$$
\mathrm{N}_{\mathrm{k}}=\frac{\lambda_{\mathrm{k}}}{\mu_{\mathrm{k}}}, \text { for } \mathrm{k}=1, \ldots, l \text { and } \mathrm{Y}_{\mathrm{j}}=\mathrm{T}_{\mathrm{i}}=0 \text {, for all } \mathrm{i}, \mathrm{j}, \mathrm{i}=1, \ldots, \mathrm{m} ; \mathrm{j}=1, \ldots, \mathrm{n} \text {. }
$$

This is the unique feasible equilibrium point whenever the removal rate (predation and death for intermediate predators, death for top predators) exceeds the value of the recruitment rate, that is, if predators are not efficient consuming prey or if the prey is not abundant enough, the predator populations go extinct. Otherwise the non-trivial equilibria in (23) are also feasible.

Recall that in a predator-prey system, unlike typical compartmental models for infectious diseases, the captured prey is removed from the original compartment but, most importantly, contributes to the recruitment of the predator that kills it. Thus, there is a net transfer of individuals from, say, prey of group $k$ to predator of group $\mathrm{j}$ and this transfer of individuals is weighted by the predator efficiencies $\alpha$ and $\beta$. 
Notice however, that we still have a conservation condition which derives from the conservation law for the mixing probabilities. In fact, if we subtract the overall losses of basal prey from the overall recruitment of intermediate predators, and the overall losses from the intermediate predators due to predation from the overall recruitment of the top predator species, and if we assume that $\alpha_{j}$, $\beta_{i}$ are unity for all $i$ and $j$, then from Eq. (21) we find

$$
\sum_{j=1}^{m} \frac{Y_{j}}{Y} \sum_{j=1}^{l} s_{k} \theta_{k j} N_{j}-\sum_{k=1}^{l} s_{k} N_{k} \sum_{j=1}^{m} \theta_{k j} \frac{Y_{j}}{Y}=0,
$$

and, likewise,

$$
\sum_{i=1}^{n} \frac{T_{i}}{T} \sum_{j=1}^{m} r_{j} Y_{j} q_{j i}-\sum_{j=1}^{m} r_{j} Y_{j} \sum_{i=1}^{n} q_{j i} \frac{T_{i}}{T}=0 .
$$

The same kind of results holds if the recruitment rate of the basal prey is changed to a logistic type of expression of the form $g_{h}(x)=\lambda_{h} \times\left(1-x / K_{h}\right)$. The same conservation property holds. However, in this case, complex periodic behavior may arise. As examples we show some numerical simulations in Figures 1-2. Figures 1-2 show the dynamics of the trophic species; that is, the total densities for each trophic level have been computed and plotted. Thus we can say that these figures represent, literally, the food web dynamics. Figure 1 shows the phase plot of a food web with, possibly, chaotic dynamics. Figure 2 shows the phase plot of a trophic web that apparently exhibits aperiodic behavior. In all phase plots only basal and intermediate levels are shown. Simulations results for the food web represented by the system (22) with logistic growth term used the parameter values $\lambda_{1}=0.07, \lambda_{2}=0.01 ; \lambda_{3}=0.02 ; d_{1}=0.011 ; d_{2}=0.02 ; d_{3}=0.03 ; \delta_{1}=\delta_{2}=\delta_{3}=0.01$. The efficiency of biomass conversion for both predator types $\left(\alpha_{i}\right.$ and $\beta_{k} \mathrm{i}=1, \ldots, \mathrm{m}$ and $\left.\mathrm{k}=1, \ldots, \mathrm{n}\right)$ is equal to 1 and $b$ and $e$ are varied ( $b$ and $e$ are the parameters needed in the expressions for $q_{i}$ and $\theta_{j}$ ). For example if $b=e=40$, and begin with three species at each level then all three basal species survive and all others go extinct. For further details see Velasco-Hernández and Castillo-Chavez (1991).

\section{4c. Relation to other models.}

The modeling framework presented in this work can be related to previous well-known models of food webs and consumer-resource interactions. For example, by taking only one species per trophic level in (21) and by defining $g[N]$ to be a logistic growth rate we essentially recover the three-species trophic web studied by Hastings and Powell (1991) since in this case the mixing probabilities are $p=1$, $q=1$ and the capture rate of each predator can be assumed to be a type II functional response. This food web may present chaotic behavior in regions of the parameter space. Likewise, by considering only two trophic levels, say $\mathrm{Y}$ and $\mathrm{N}$ in Eq.(21), we end up with a resource-consumer model of the type described by Tilman (1982) in which consumers compete for abiotic resources, sharing these resources according to the mixing probabilities $\mathrm{q}_{\mathrm{ji}}$. In our case, however, these probabilities are not constant. The simplest possible case occurs when we assume identical searching and handling times for all consumers. Here $q_{j i}$ describes a resource-consumer system where the resource share of each consumer is proportional to its frequency in the population.

4d. Remarks. Models of food webs.can be divided into two categories: classical, or static, and dynamic. Dynamic models include those of Hastings and Powell (1991) and Tilman (1991), and they are the main topic of this section. Classical models include those that attempt to describe, from a statistical point of view, characteristic patterns common to sets of food webs. The cascade model of Cohen et al. (1990) provides a successful example of a classical or static model. It describes the plausible behavior of the population densities of trophically related species.

The concept of trophic species on which 'static' food web models are based is not a natural biological class but rather a theoretical classification. It is an equivalence class made of those organisms, regardless of the species to which they belong, that share the same predators and the same prey. Thus, when one tries to define a trophic species from data sources of error exist associated with the identification of common predators and common prey. The applicability of the concept of trophic species to the field of applied ecology depends heavily on the ability of experimentalists to eliminate errors in the classification process. This problem is not unique to the study of food web dynamics; it is quite common in the biological and social sciences. Once the researcher establishes what is a weak (negligible) interaction and what is a strong one, the trophic level of each organism is determined. 'Towever, as the study of Polis (1991) shows, it is difficult to decide what is a weak and what is a strong interaction in a trophic web.

Moreover, the existence of omnivory makes it difficult to justify the classification of organisms 
into trophic species. This is particularly important if one is interested in the population dynamics of the web. Omnivory implies a very diverse diet with some items being more frequent than others (a matter of taste, opportunity, or chance), and if a large share of a predator's diet is made up of rare organisms, their neglect on the basis of weak or strong interactions may be misleading. Consequently, the trophic structure dynamics of the food web cannot be defined exclusively in terms of strong interactions, but rather through a 'distribution' of interactions that reflects the composition of an organism's diet. In a recent study Paine (1992) concludes that in an species-rich herbivore guild there are mainly weakly negative or positive interactions with only a few strong negative ones.

A trophic web may be better described from a dynamical point of view if the description centers on what resources are used and the way these resources are used, that is, if the description is centered on guilds of species. A trophic web is a model of the interrelations between species that share common resources which have been shaped by natural selection through various mechanisms such as diffuse co-evolution (Maddox and Root, 1990). In fact, the mechanism of switching or apostatic selection has been recognized as one of the factors that may promote diversity in prey populations (Greenwood 1984; Levin and Segel, 1982).

For the simple three-level food web discussed here, we have shown that the conservation principle of the mixing probabilities (cf. Definition 1) allows us to structure predator-prey interaction in terms of two main factors: the probability that a predator encounters its prey, and the conditional probability of capturing and eating the prey once it has been found. Moreover, the use of the mixing framework described in this work constitutes an approach to the modeling of switching predator behavior, of frequency-dependent mechanisms in prey selection, and interaction strength in food webs.

We have also shown that the conservation law of the mixing probabilities translates into a conservation property in the predator capture and consumption rates, and the prey capture and removal (through predation) rate, as shown in Eq. (15a) and (15b). Eq. (13) is essentially a consumer-resource model where the probability that during a unit time, an individual in consumer species $j$ captures and consumes an individual in species $i$ is not constant but determined by the frequency of predators and prey involved in the interaction, and the preference or risk of each predator or prey type respectively. In fact, only when the mixing probabilities are constant, and the ratio of resource of type $k$ numbers to total consumer numbers is equal to the density of resource prey of type k, do we recover a Lotka-Volterra (Kolmogorov type, Brauer, 1976) model.

The models of food web dynamics based on our framework can be used, for example, to study the time evolution of any given initial food web configuration. It is possible to assign different 'strengths' to the interactions in the web through the initial contact rates satisfying conservation relations as the one shown in (22b), and then follow through time the fate of the individual species.

\section{APPLICATION TO MODELS FOR VECTOR TRANSMITTED DISEASES.}

Our efforts to contribute to the understanding of the relative effects of different mechanisms on the dynamics of the HIV/AIDS epidemic constitute the driving force behind the development of mixing/contact structures that we have discussed in this article, and consequently their application has been in the context of STD's. In this section, we outline the use of our framework in the context of vector-transmitted diseases.

\section{5a. Host-Vector Mixing Frameworks.}

Mathematical models have played a very important role in the history and development of vector-parasite epidemiology. Sir Ronald Ross in 1911 developed a theory for parasite transmission mechanism while engaged in the study of malaria. He developed a simple mathematical model that provided him with the concept of transmission threshold. Later on, Macdonald (1957), based on the work of Ross, developed a new model from which he extracted the concept of vectorial capacity. The involvement of mathematical models in epidemiological theory has persisted along the years. In particular we mention the mathematical model developed for the evaluation of control measures for malaria in the Garki Project (Molineaux and Gramiccia, 1980). This model was developed based on an extensive research on the transmission of malaria in Northern Nigeria. It uses the theory and results developed by Ross and Macdonald. Despite the construction of new models (e.g. Aron and May, 1982; Rogers, 1988; Pacala and Dobson, 1990; Dietz, 1988b), all estimates and assumed mechanisms for parasite transmission from vector to host still rely on the assumptions implicit in the definition of vectorial capacity as presented by Macdonald (1957). It is estimated in order to 
understand and evaluate the strength and effectiveness of vectors for the transmission of parasites.

Transmission in vector-transmitted diseases depends on the life history of the vector species. Malaria and dengue, for example, are transmitted by mosquitoes of several species including Aedes $s p p$. for dengue and Anopheles spp. for malaria. Chagas' disease is transmitted by triatominae bugs of various genuses, e.g. Triatoma spp., Rhodnius spp., and Pastrongylus spp. In malaria and dengue, transmisson of the parasite to human hosts involves only adult individuals since the larval stages are aquatic and have a completely different ecological niche. In Chagas' disease, however, the vector is a triatomine bug with 5 nymphal stages preceding reproductive maturity. All of them are hematophagous and all of them may be involved in the transmission process (Zeledon and Rabinovich, 1981). Thus when speaking about general models for vector-parasite-host interactions we must consider the age and stage structure of the vector population.

\section{5b. Vectorial Capacity}

The factors affecting transmission by the appropriate vector stages according to Molineaux (1988) are: (1) density of the vectors in relation to human hosts; (2) the effectivennes of the vector in acquiring and maturing the infection after feeding on an infective subject; (3) the frequency with which the vector takes a blood meal and the fraction of these blood meals taken on human hosts; (4) the duration of the parasite incubation period in the vector; (5) the longevity of the vector. Most of the above factors are included in the formula for the vectorial capacity defined as the capacity of the vector population to transmit the disease in terms of the potential number of secondary inoculations originating per unit time from an infective person (Molineaux, 1988; Molineaux et al., 1988). A formula for the vectorial capacity is derived form the concept of basic reproductive rate (not a rate) proposed by Macdonald (1957):

$$
\mathrm{C}=\frac{\mathrm{ma}^{2} \mathrm{p}^{\mathrm{n}}}{-\ln \mathrm{p}}
$$

where $\mathbf{C}$ denotes the vectorial capacity, $m$ the number of vectors per human host, a the number of blood meals taken on a human host per vector per day (biting rate), $p$ the proportion of vectors surviving per day, and $n$ the length in days of the parasite incubation period in the vectors. Formula (25) is species specific since, for example, $\mathbf{n}$ varies from species to species.

If we denote by $R_{0}$ the basic reproductive number or ratio, then we have that

$$
R_{0}=\frac{C}{r}
$$

where $r^{-1}$ denotes the expected duration of infectivity (Molineaux. 1988). If we define $\mu$ as the death rate then $\mathrm{p}=\mathrm{e}^{-\mu}$ and

$$
\mathrm{C}=\frac{\mathrm{ma}^{2} \mathrm{e}^{-\mu \mathrm{n}}}{\mu}
$$

then $R_{0}=\frac{C}{\mu r}$, the form used by Aron and May (1982) in their models of Malaria transmission.

The assumptions on which (25) is based are, according to Molineaux (1988): (a) the vector is fully effective in acquiring and maturing the infection. This amounts to ignoring variability in susceptibility by the vector species; (b) vectors die at constant rate, independent of age, and senescence is ignored; (c) longevity is unaffected by the infection; (d) the probability of feeding on hosts is unaffected by the number of previous meals or by differences in host type, and (e) parasite presence does not affect preference by vectors (Dye, 1990).

Some of the important factors neglected in Formula (25) were described by Ribeiro, Rossignol, and Spielman (1985) in their study on the blood finding strategy of Aedes aegipty and its interaction with the parasite Plasmodium gallinaceum. The probing behaviour by Aedes aegipty is complex and involves periodically repeated probing while searching for a blood meal (Ribeiro et al., 1985): new attempts to feed depend on the success of the previous search. During each search the probability of feeding success may be interpreted as a function of the blood vessels on the skin. There is some evidence that the probability of desisting from feeding increases linearly with time while decreasing as a function of the previous number of attempts. Ribeiro et al. (1985) conclude that the dependence of the probability of feeding success on the density of blood vessels implies a preference for infected hosts since parasites induce an increase in the availability of blood vessels (see also Molyneaux and Jeffreries, 1986).

Feeding and probing behaviour of hematophagous arthropods may change during feeding depending on the infected status of the host, and this fact, according to Dye (1990), makes direct estimation of the vectorial capacity impossible. He argues that it is better to estimate the relative 
vectorial capacity before and after a control measure is applied.

In Chagas' disease, the feeding and developmental cycle can be broken into clear stages (see the study on the population of Rhodnius prolixus, Friend et al., 1965). Trypanosoma cruzi is transmitted to susceptible hosts by contamination rather than by injection during biting. Triatomines urinate and defecate during or after a blood meal (Zeledon and Rabinovich, 1981), and this excretory behavior impacts the transmission dynamics of the parasite. Infective forms of $T$. cruzi go in the feces and are able to penetrate wounds or soft tissue-around the eyes and the mouth-infecting the host. The probability of infection increases with the duration of a blood meal (Zeledon and Rabinovich, 1981). Hess and Hayes (1970) have explored the potential of domestic animals to attract zoophilic species of mosquito (Culex tarsalis and other species of the same genus). It has been established that preferences do exist among vector populations in the selection of hosts; however, host preference in the field depends not only on the vector preferences but on the density and relative abundance of host types.

These results indicate that a careful modelling of the process of acquisition of blood meals by vectors is necessary in order to obtain a better estimate of the transmission probability. Searching and handling times must be explicitly considered as well as the functional form of the dependence of the transmission probability on these parameters. We cannot proceed to use formula (25) when the vector does not transmit the disease by biting, as in the case of Chagas' disease, when there is more than one stage involved in the transmission process (Schofield, 1982; Zeledon and Rabinovich, 1981), or when handling, search times, host-preference, and frequency and density dependent effects are important.

\section{5c. Contact structure.}

The need for further theoretical work is therefore quite evident. We re-interpret the contact structure for frequency-dependent predation, completed in Section 4, in the context of host-vector interactions. We hope that our approach will provide a useful framework on which the questions raised may be systematically addressed.

Assume that vectors as well as hosts are subdivided into groups according to some variable of interest (geographical location, susceptibility to infection, species, etc.), and denote by $\mathrm{C}_{\mathrm{ij}}$ the average number of contacts per day that vectors of group $i$ have with hosts in group $j$. Consequently ( for $i=$ $1, \ldots, M$ and $j=1, \ldots, N)$ we must have

$$
V_{i}(t) C_{i j}(t)=H_{j}(t) C_{j i}(t),
$$

where $V_{i}(t)$ denotes the number of vectors of type $i$ at time $t$ and $H_{j}(t)$ denotes the number of hosts of type $j$ at time $t$.

Let $\tau_{i}$ denote the total relative average time spent foraging and $\sigma_{i j}$ the average handling time spent on hosts of group $j$ by a typical vector of type $i$. Then, as before,

$$
\tilde{\sigma}_{i}:=\sum_{k=1}^{N} \sigma_{i k} C_{i k},
$$

denotes the mean per capita handling time of vectors of group $i$, and the searching time available for vectors of type $i$ to make contacts, that is, to find suitable hosts, is $\tau_{i} \tilde{\sigma}_{i}$. Therefore, the average number of contacts that a typical vector of type $i$ makes with hosts of type $j$ per unit time is

$$
\mathrm{C}_{\mathrm{ij}}=\left(\tau_{\mathrm{i}}-\tilde{\sigma}_{\mathrm{i}}\right) \beta_{\mathrm{ij}} \mathrm{H}_{\mathrm{j}} \text {, }
$$

where $\beta_{\mathrm{ij}}$ denotes the rate of successful contacts, i.e., the actual biting rate of vectors of type $i$ on hosts of type $j$. In many situations it is reasonable to assume that $\beta_{i j}$ is a function of the total number of vectors of any type feeding at time $t$ on hosts of type $j$. If $\Pi_{j}$ denotes the average number of vectors of any type feeding on a host in group $j$, and if we assume that vectors are less efficient at biting very popular hosts (that is, hosts with many vectors feeding on them), then as a first approximation we have that

$$
\beta_{\mathrm{ij}}=\frac{\mathbf{r}_{\mathrm{ij}}}{\Pi_{\mathbf{j}}}
$$

where the matrix $\left(\mathrm{r}_{\mathrm{ij}}\right)$ can be interpreted as the matrix of maximum biting rates at low vector densities. We impose the condition $r_{i j}=r_{j i}$ for all $1 \leq \mathrm{i} \leq \mathrm{M}, 1 \leq \mathrm{j} \leq \mathrm{N}$, and set $\mathrm{r}_{\mathrm{ji}}=0$ otherwise. Substituting (29-30) into (28) one obtains ai before

$$
\tilde{\sigma}_{\mathrm{i}}=\left(\tau_{\mathrm{i}}-\tilde{\sigma}_{\mathrm{i}}\right) \sum_{\mathrm{k}=1}^{\mathrm{N}} \sigma_{\mathrm{ik}} \mathrm{r}_{\mathrm{ik}} \mathrm{m}_{\mathrm{k}},
$$


where $m_{k}=\frac{H_{k}}{\Pi_{k}}$ is the ratio of hosts of kth type to vectors of any type feeding on a host of type $k$.

If $p_{i j}$ denotes the proportion of effective contacts between vectors of type $i$ and hosts of type $j$, then one easily sees (as in Section 2) that

$$
p_{i j}=\frac{r_{i j} m_{j}}{\sum_{j=1}^{N} r_{i j} m_{j}}
$$

Similarly, if $q_{j i}$ denotes the proportion of effective contacts between hosts of type $j$ with vectors of type $\mathrm{i}$ then

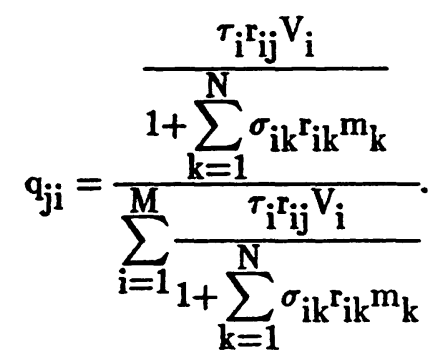

Therefore the matrix $\left(\mathrm{p}_{\mathrm{ij}}, \mathrm{q}_{\mathrm{ji}}\right)$ is a mixing contact matrix; that is, it satisfies properties (di)-(div). Solutions (31-32) are not Ross-solutions, however, they become Ross solutions by requiring that $\mathbf{r}_{\mathrm{ij}}=$ $r$ for all indices (see Section 4, Equations $15 \mathrm{a}$ and $15 \mathrm{~b}$ ).

Remark 2. From Equations (15a and $15 \mathrm{~b}$ ) we see that the mixing of susceptible hosts depends on the ratio $\mathrm{m}_{\mathrm{j}}$-measuring how the host population is allocated to each vector type-while the mixing of susceptible vectors depends on the foraging time invested in 'capturing' a host. In this sense, solutions (15a) and (15b) and, more generally solutions (31) and (32), allow for the possibility of modelling asymetric forms of transmission as those discussed in relation to Chagas' disease (see Velasco-Hernández, 1991).

In a classical contact epidemic model, which is quite appropriate for host-vector interactions, the incidence rate for infective hosts, or the number of newly infected hosts per unit time, has the general form

$$
S_{h_{j}} \sum_{i=1}^{M} p_{i j} g_{i} \frac{I_{v_{i}}}{S_{v_{i}}+I_{v_{i}}}
$$

where $g_{i}$ is a parameter that measures the infectivity of vectors of type $i, S_{v_{i}}$ and $I_{v_{i}}$ are the susceptible and infective subpopulations respectively of vectors of type $i$, and $S_{h}{ }_{h}$ is the susceptible host population of type $\mathrm{j}$. Similarly, the incidence rate of infected vectors has the general form

$$
S_{v_{i}} \sum_{i=1}^{M} q_{j i} f_{j} \frac{I_{h_{j}}}{S_{h_{j}}+I_{h_{j}}}
$$

where $f_{j}$ is a parameter that measures the infectivity of hosts of type $j$, and $S_{h_{j}}$ and $I_{h_{j}}$ are the susceptible and infective host populations respectively. The full equations are then $\dot{j}_{\text {readily }} \dot{b}_{\text {written. }}$ Some preliminary work using these equations has begun to be carried out by Velasco-Hernández (1991).

\section{CONNECTIONS BETWEEN DETERMINISTIC AND STOCHASTIC APPROACHES}

Classical deterministic models for the sexual spread of STD's such as gonorrhea among heterosexual populations can be found in Hethcote and Yorke (1984) while classical and pairformation models under a unified mixing framework for the spread of STD's can be found in Blythe et al. (1991), and references therein. A stochastic version of one of the deterministic models found in Blythe et al. (1991) is provided below. This formulation uses the modeling approach common to interacting particle systems (for details see Luo and Castillo-Chavez 1991, 1992). Hence, it has great generality and flexibility. 


\section{General Notation}

Let $X=\{0,1, \cdots, L\} \times\{0,1\} \times\{0,1, \cdots, N\} \times\{0,1\} \backslash\{0\} \times\{0,1\} \times\{0\} \times\{0,1\}$ and consider the stochastic process $\xi_{t}: X \rightarrow\{0,1,2, \cdots\}, t \geq 0$. For $x=(i, \mu ; j, \nu) \in X$, our interpretation of this process is as follows:

(1) The labels $\mu$ and $\nu$ represent the eepidemiological status of the individuals. Specifically, $0 \equiv$ susceptible and $1 \equiv$ infected. The labels $i$ and $j$ represent groups of males and females.

(2) For $\mathrm{i}>0$ and $\mathrm{j}>0, \xi_{t}(\mathrm{i}, \mu ; \mathrm{j}, \nu)$ gives the number of pairs where the male is of the $\mathrm{i}$-th type and has epidemiological status $\mu$ and the female is of the $\mathrm{j}$-th type and has status $\nu$ at time $\mathrm{t}$;

(3) Singles are labeled by triplets. However, to keep the domain fixed we use four coordinates and set either $i$ or $j$ equal to zero. Specifically, if $i>0$ and $j=0$, then $\xi_{t}(i, \mu ; 0) \equiv \xi_{t}(i, \mu ; 0,0)$ $\equiv \xi_{t}(i, \mu ; 0,1)$ denotes the number of single males with status $\mu$ in the $\mathrm{i}$-th subpopulation at time t. Similarly, if $\mathrm{i}=0$ and $\mathrm{j}>0$, then $\xi_{\mathrm{t}}(0 ; \mathrm{j}, \nu) \equiv \xi_{\mathrm{t}}(0,0 ; \mathrm{j}, \nu) \equiv \xi_{\mathrm{t}}(0,1 ; \mathrm{j}, \nu)$ denotes the number of single females with status $\nu$ in the $j$-th subpopulation at time $t$.

Let $S \equiv\{0,1,2, \cdots\}^{X}$ and let $c: S \times S \rightarrow(0, \infty)$ be a real-valued function-the flip rate-to be specified later. We view $\left\{\xi_{t}: t \geq 0\right\}$ as an S-valued Markov process with flip rate $c\left(.\right.$, .), i.e., if $\xi_{t}=\xi$ for some $t \geq 0$ then $c(\xi, \eta)$ denotes the instantaneous rate at which $\xi_{t}$ may change to the state $\eta$. The generator of this process is

$$
\Omega f(\xi)=\sum_{\eta} c(\xi, \eta)(f(\eta)-f(\xi))
$$

where $f$ is a continuous function on $S$. Thus,

$$
\frac{d}{d t} E f\left(\xi_{t}\right)=E \sum_{\eta} c(\xi, \eta)\left(f(\eta)-f\left(\xi_{t}\right)\right)
$$

We further assume the existence of an underlying mixing/pair-formation matrix $\left(p_{i j}(\xi), q_{i j}(\xi)\right)$ of the type described in Section 2. To specify the flip rates we use the following notation. For $\xi \in S, A \subset X$, $B \subset X$ and $A \cap B=0$, we define $\xi_{B}^{A} \in S$ as

$$
\xi_{B}^{A}(x)=\left\{\begin{array}{ll}
\xi(x)+1 & \text { if } x \in A \\
\xi(x)-1 & \text { if } x \in B \\
\xi(x) & \text { otherwise }
\end{array} .\right.
$$

If we change the notation slightly and now use the letters $m$ and $f$ to denote the parameters associated with uninfected males and females and $M$ and $F$ to denote those associated with infected males and females then one defines the flip rate $c(.,$.$) as follows (here \gamma, \delta$, and $\sigma$ are constant parameters):

(a) Pair-formation

For $\mathrm{i}>0, \mathrm{j}>0$,

$$
\mathrm{c}\left(\xi, \xi \xi_{(\mathrm{i}, \mu ; 0),(0 ; \mathrm{j}, \nu)}^{(\mathrm{i}, \mu \mathrm{j}, \nu)}\right)=\mathrm{b}_{j}^{f}(\xi) \xi(0 ; \mathrm{j}, \nu) \mathrm{p}_{\mathrm{ji}}^{\mathrm{f}}(\xi) \frac{\xi(\mathrm{i}, \mu ; 0)}{\xi(\mathrm{i}, \mu ; 0)+\xi(\mathrm{i}, 1-\mu ; 0)} .
$$

(b) Pair-dissolution ( $\sigma$ denotes the pair-dissolution rate)

For $\mathrm{i}>0, \mathrm{j}>0$,

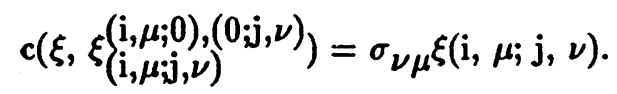

(c) Transmission ( $\delta$ denotes the transmission coefficient-transmission may occur only while paired) For $\mathrm{i}>0, \mathrm{j}>0$,

$$
c\left(\xi, \xi\left(\begin{array}{l}
\mathrm{i}, 0 ; \mathrm{j}, 1,1) \\
(\mathrm{i}, 1)
\end{array}\right)=\delta_{\mathrm{F}} \xi(\mathrm{i}, 0 ; \mathrm{j}, 1), \mathrm{c}\left(\xi, \xi_{(\mathrm{i}, 1 ; j, 0)}^{(\mathrm{i}, 1 ; \mathrm{j}, 1)}\right)=\delta_{\mathrm{M}} \xi(\mathrm{i}, 1 ; \mathrm{j}, 0) .\right.
$$

(d) Recovery ( $\gamma$ denotes the recovery rate)

For $\mathrm{i}>0, \mathrm{j}>0$,

$$
\begin{aligned}
& c\left(\xi, \xi\left(\begin{array}{l}
\mathrm{i}, 0 ; \mathrm{j}, 0) \\
(\mathrm{i}, 0 ; \mathrm{j}, 1)
\end{array}\right)=\gamma_{\mathrm{F}} \xi(\mathrm{i}, 0 ; \mathrm{j}, 1), \mathrm{c}\left(\xi, \xi\left(\begin{array}{l}
\mathrm{i}, 0 ; \mathrm{j}, 0) \\
\mathrm{i}, 1 ; \mathrm{j}, 0)
\end{array}\right)=\gamma_{\mathrm{M}} \xi(\mathrm{i}, 1 ; \mathrm{j}, 0)\right.\right. \\
& \mathrm{c}\left(\xi, \xi\left(\begin{array}{l}
\mathrm{i}, 1 ; \mathrm{j}, 0) \\
(\mathrm{i}, 1 ; \mathrm{j}, 1)
\end{array}\right)=\gamma_{\mathrm{F}} \xi(\mathrm{i}, 1 ; \mathrm{j}, 1), \mathrm{c}\left(\xi, \xi\left(\begin{array}{l}
\mathrm{i}, 0 ; \mathrm{j}, 1) \\
\mathrm{i}, 1 ; \mathrm{j}, 1)
\end{array}\right)=\gamma_{\mathrm{M}} \xi(\mathrm{i}, 1 ; \mathrm{j}, 1)\right.\right.
\end{aligned}
$$


while for single infected individuals we have

$$
c\left(\xi, \xi\left(\begin{array}{l}
\mathrm{i}, 0 ; 0) \\
\mathrm{i}, 1 ; 0)
\end{array}\right)=\gamma_{\mathrm{M}} \xi(\mathrm{i}, 1 ; 0), \mathrm{c}\left(\xi, \xi \xi_{(0 ; j, 1)}^{(0 ; j, 0)}\right)=\gamma_{\mathrm{F}} \xi(0 ; \mathrm{j}, 1)\right.
$$

furthermore, for $\mathrm{i}>0, \mathrm{j}>0$ we have the combined recovery rate

$$
c\left(\xi, \xi(\mathrm{i}, 1 ; j, 1)=\gamma_{F M} \xi(i, 1 ; j, 1) .\right.
$$

(e) Removal ( $\mu$ denotes the removal rate from sexual activity)

For $\mathrm{i}>0, \mathrm{j}>0, \mu, \nu$,

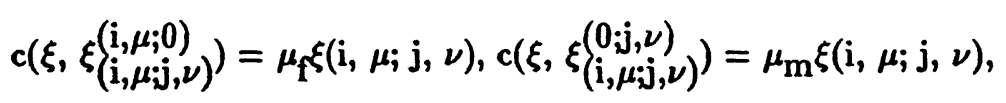

while for the removal rate of single individuals we have that

$$
c\left(\xi, \xi_{(i, \mu ; 0)}\right)=\mu_{m} \xi(i, \mu ; 0), c\left(\xi, \xi_{(0 ; j \nu)}\right)=\mu_{\mathrm{f}} \xi(0 ; j, \nu) .
$$

(f) Recruitment (all recruited individuals are susceptible)

For $\mathrm{i}>\mathbf{0 , j}>\mathbf{0}$,

$$
c\left(\xi, \xi^{(i, 0 ; 0)}\right)=\Lambda_{i}^{m}, c\left(\xi, \xi^{(0 ; j, 0)}\right)=\Lambda_{j}^{f}
$$

(g) Other:

For any other $\eta \neq \xi$, we assume $c(\xi, \eta)=0$ and $c(\xi, \xi)=-\sum_{\xi \neq \eta} c(\xi, \eta)$.

(More details are found in Luo and Castillo-Chavez, 1991, 1992).

As the time $t$ changes, singles may form pairs, pairs may dissolve, and the disease may be transmitted (usually only within clearly specified pairings). The system $\left\{\xi_{t}\right\}$ consists of a series of changing elements in the set $X$, the set of all functions on $S$. The dynamics of the system is described by the rates at which the system changes. These rates are given as a set of nonnegative numbers $\{c(\xi, \eta): \xi \neq \eta, \xi, \eta \in X\}$. Specifically, each $c(\xi, \eta)$ is the rate at which the system changes from $\xi$ to $\eta$, that is,

$$
\mathrm{P}\left(\xi_{t+h}=\eta / \xi_{t}=\xi\right)=c(\xi, \eta) \mathrm{h}+0(\mathrm{~h}), \forall t \geq 0 .
$$

The deterministic model that corresponds to the above stochastic model in the context of a STD such as gonorrhea (susceptible $\rightarrow$ infected $\rightarrow$ susceptible) but that incorporates couples (transmission can only occur in a couple where one of the partners is infected) is described below (for more details see Blythe et al. 1991).

Consider a population of sexually active heterosexual individuals divided into subpopulations by such factors as sex, race, socio-economic background, and average degree of sexual activity. There are $N^{\mathrm{f}}$ female and $N^{\mathrm{m}}$ male subpopulations, each divided into two epidemiological classes for single individuals: $f_{\mathrm{j}}(t)$ and $m_{\mathrm{i}}(t)$ (single susceptible females and males, at time $t$ ), and $F_{\mathrm{j}}(t)$ and $M_{\mathrm{i}}(t)$ (single infected females and males), all for $j=1, \ldots, N^{\mathrm{f}}$ and $i=1, \ldots, N^{\mathrm{m}}$. Hence the sexually-active single individuals of each sex and each subpopulation are given by $T_{\mathrm{j}}^{\mathrm{f}}=f_{\mathrm{j}}+F_{\mathrm{j}}$ and $T_{\mathrm{i}}^{\mathrm{m}}=m_{\mathrm{i}}+M_{\mathrm{i}}$. The epidemiological classes for pairs are given by $\pi_{\mathrm{ji}}^{\mathrm{fm}}, \pi_{\mathrm{ji}}^{\mathrm{Fm}}, \pi_{\mathrm{ji}}^{\mathrm{M}}, \pi_{\mathrm{ji}}^{\mathrm{FM}}$, which are respectively the numbers of pairs of $f$-with- $m, F$-with- $m, f$-with- $M$, and $F$-with- $M$ individuals. Transmission can only occur among those individuals in pair types $\pi_{\mathrm{ji}}^{\mathrm{Fm}}$ or $\pi_{\mathrm{ji}}^{\mathrm{fM}}$. Since $\pi_{\mathrm{ji}}^{\mathrm{fM}}=\pi_{\mathrm{ij}}^{\mathrm{Mf}}$, we need only consider four types of pairs. We assumed that the transmission probability per unit time is constant within each pair containing one infected individual. We let $\delta_{M}$ and $\delta_{F}$ be the rates for male-to-female and female-to-male transmission, respectively. The per capita recovery rates are $\gamma_{M}$ and $\gamma_{F}$ for infected males and infected females, respectively, when their partner is uninfected. When both partners are infected ( $F$-with- $M$ pairs), simultaneous treatment of both is the norm for gonorrhea, so we incorporate "combined" recovery rate $\gamma_{\mathrm{FM}}$, with both parties moving directly to the $f$-with- $m$ (no infection) pair type. The per capita dissolution rates are $\sigma_{\mathrm{fm}}, \sigma_{\mathrm{fM}}, \sigma_{\mathrm{Fm}}$, and $\sigma_{\mathrm{FM}}$ for the different types of pairs, and the per capita removal rates from sexual activity due to death or other causes are $\mu_{\mathrm{f}}$ and $\mu_{\mathrm{m}}$ for all females and all males respectively. Let $\Lambda_{\mathrm{j}}^{\mathrm{f}}$ and $\Lambda_{\mathrm{i}}^{\mathrm{m}}$ denote the "recruitment" rates (assumed constant) of single (assumed uninfected) individuals in the female and male populations 
respectively. We use the notation

$$
p_{j i}^{x m} \equiv \frac{m_{i}}{M_{i}+m_{i}} p_{j i}^{f}, \quad p_{j i}^{x M} \equiv \frac{M_{i}}{M_{i}+m_{i}} p_{j i}^{f}, \quad p_{i j}^{y f} \equiv \frac{f_{i}}{F_{i}+f_{i}} p_{i j}^{m}, \quad p_{i j}^{y F} \equiv \frac{F_{i}}{F_{i}+f_{i}} p_{i j}^{m},
$$

( $x=f$ or $F$ and $y=m$ or $M$, for $i=1, \ldots, N^{\mathrm{m}}$ and $j=1, \ldots, N^{\mathrm{f}}$ ) for the fraction of pair-formations between the specified sub-groups $\left(i\right.$ and $j$ ) which are of given infection status; for example, $p_{\mathrm{ji}}^{\mathrm{fm}}$ and $p_{\mathrm{ji}}^{\mathrm{fM}}$ give the fractions involving uninfected $\left(m_{\mathrm{i}}\right)$ and infected $\left(M_{\mathrm{i}}\right)$ males respectively. Then the gonorrhea pair formation/dissolution model is

$$
\begin{aligned}
& \frac{d f_{j}}{d t}=\Lambda_{j}^{f}+\gamma_{F} F_{j}+\left[\mu_{M}+\sigma_{f M}\right] \sum_{i=1}^{N^{m}} \pi_{j i}^{f M}+\left[\mu_{m}+\sigma_{f m}\right] \sum_{i=1}^{N^{m}} \pi_{j i}^{f m}-\left[C_{j}^{f}(t)+\mu_{f}\right] f_{j}, \\
& \frac{d F_{j}}{d t}=\left[\mu_{m}+\sigma_{F m}\right] \sum_{i=1}^{N^{m}} \pi_{j i}^{F m}+\left[\mu_{m}+\sigma_{F M}\right] \sum_{i=1}^{N^{m}} \pi_{j i}^{F M}-\left[C_{j}^{f}(t)+\gamma_{F}+\mu_{i}\right] F_{j} \text {, } \\
& \frac{d m_{i}}{d t}=\Lambda_{i}^{m}+\gamma_{M} M_{i}+\left[\mu_{r}+\sigma_{F m}\right] \sum_{j=1}^{N^{f}} \pi_{j i}^{F m}+\left[\mu_{r}+\sigma_{f m}\right] \sum_{j=1}^{N^{f}} \pi_{j i}^{f m}-\left[C_{i}^{m}(t)+\mu_{m}\right] m_{i} \text {, } \\
& \frac{d M_{i}}{d t}=\left[\mu_{r}+\sigma_{\mathrm{gM}}\right] \sum_{j=1}^{N^{f}} \pi_{j i}^{f M}+\left[\mu_{r}+\sigma_{F M}\right] \sum_{j=1}^{N} \pi_{j i}^{F M}-\left[C_{i j}^{m}(t)+\gamma_{M}+\mu_{M}\right] M_{i} \text {, } \\
& \frac{d \pi_{j i}^{f m}}{d t}=C_{j}^{f}(t) p_{j i}^{f m} f_{j}+\gamma_{M} \pi_{j i}^{f M}+\gamma_{F} x_{j i}^{F m}+\gamma_{F M} \pi_{j i}^{F M}-\left[\mu_{\Gamma}+\mu_{m}+\sigma_{f m}\right] x_{j i}^{f m}, \\
& \frac{d \pi_{j i}^{F m}}{d t_{m}^{F}}=C_{j}^{f}(t) p_{j i}^{F m} F_{j}+\gamma_{M} \pi_{j i}^{F M}-\left[\mu_{\mathrm{f}}+\mu_{m}+\sigma_{F m}+\delta_{F}+\gamma_{F}\right] \pi_{j i}^{F m}, \\
& \frac{d \pi_{j i}^{\mathrm{fM}}}{d t \mathrm{FM}}=C_{\mathrm{j}}^{f}(\mathrm{t}) \mathrm{p}_{\mathrm{ji}}^{\mathrm{fM}} \mathrm{f}_{\mathrm{j}}+\gamma_{\mathrm{F}} \pi_{\mathrm{ji}}^{\mathrm{FM}}-\left[\mu_{\mathrm{f}}+\mu_{\mathrm{m}}+\sigma_{\mathrm{fM}}+\delta_{\mathrm{M}}+\gamma_{\mathrm{M}}\right] \pi_{\mathrm{ji}}^{\mathrm{fM}} \text {, } \\
& \frac{\mathrm{d} \pi_{\mathrm{ji}}^{\mathrm{FM}}}{\mathrm{dt}}=\mathrm{C}_{\mathrm{j}}^{\mathrm{f}}(\mathrm{t}) \mathrm{p}_{\mathrm{ji}}^{\mathrm{FM}} \mathrm{F}_{\mathrm{j}}+\delta_{\mathrm{F}} \pi_{\mathrm{ji}}^{\mathrm{Fm}}+\delta_{\mathrm{M}} \pi_{\mathrm{ji}}^{\mathrm{fM}}-\left[\mu_{\mathrm{f}}+\mu_{\mathrm{m}}+\sigma_{\mathrm{FM}}+\gamma_{\mathrm{M}}+\gamma_{\mathrm{F}}+\gamma_{\mathrm{FM}}\right] \pi_{\mathrm{ji}}^{\mathrm{FM}} \text {, }
\end{aligned}
$$

with initial conditions $f_{\mathrm{j}}(0)>0, m_{\mathrm{i}}(0)>0, \pi_{\mathrm{ji}}^{\mathrm{fm}}(0)=0, \pi_{\mathrm{ji}}^{\mathrm{fM}}(0)=0, \pi_{\mathrm{ji}}^{\mathrm{Fm}}(0)=0, \pi_{\mathrm{ji}}^{\mathrm{FM}}(0)=0$, and at least one of the $F_{\mathrm{j}}(0)$ and $M_{\mathrm{i}}(0)$ greater than zero (for $i=1, \ldots, N^{\mathrm{m}}$ and $j=1, \ldots N^{\mathrm{f}}$ ). We invoke the results of Kurtz $(1970,1971)$ and conclude that for large populations the deterministic and stochastic models will have the same asymptotic behavior.

\section{6a. Simulations of the process $\left\{\xi_{t}: t \geq 0\right\}$.}

The general approach for simulating jump Markov processes is as follows. From the construction of the flip rates we know that

$$
\mathrm{c}(\xi)=\sum_{\eta \in \mathrm{S}} \mathrm{c}(\xi, \eta)<\infty
$$

Hence, if we let the sequence $0=\rho_{0}<\rho_{1}<\rho_{2}<\cdots$ denote the jump times of the process. Then $\tau_{\mathrm{n}}$ $=\rho_{\mathrm{n}}-\rho_{\mathrm{n}-1}$ has an exponential distribution with rate $\mathrm{c}\left(\xi_{\sigma_{\mathrm{n}-1}}\right)$. We can simulate the process using
the following procedure:

(i) Set the initial state $\xi_{0}$ and assume that a sequence of n jump times $0=\sigma_{0}<\sigma_{1}<\cdots<\sigma_{\mathrm{n}}$ and their corresponding states $\xi_{\sigma_{i}}, 1 \leq \mathrm{i} \leq \mathrm{n}$ have been determined.

(ii) Get $\tau_{\mathrm{n}+1}$ from $\exp \left\{\mathrm{c}\left(\xi_{\sigma_{\mathrm{n}}}\right)\right\}$ and let $\sigma_{\mathrm{n}+1}=\sigma_{\mathrm{n}}+\tau_{\mathrm{n}}$.

(iii) Set $\xi_{\sigma_{n+1}}=\eta \quad$ with probability $\quad \frac{c\left(\xi_{\sigma_{n}}, \eta\right)}{c\left(\xi_{\sigma_{n}}\right)}$.

(iv) Define $\xi_{\mathrm{t}}=\xi_{\sigma_{\mathrm{n}}}$ for $\sigma_{\mathrm{n}} \leq \mathrm{t}<\sigma_{\mathrm{n}+1}$.

We proceed to simulate the above stochastic model in a very special situation. We assume that the infection rates $\delta_{\mathrm{M}}$ and $\delta_{\mathrm{F}}$ are equal to zero or equivalently that there are no infected individuals in the population. Hence, we are simulating a purely demographic model. Individuals form and dissolve pairs. There is constant recruitment and we have individuals of several (economic, social, etc.) types. The simulation (described below) will have as its average dynamics the corresponding deterministic dynamics. However, the stochastic version allows for the study the changes of the variance as a function of time.

A 10,000 -realization run vas allowed to simulate the process up to time $t=2.0$, and a 1,000 realization run was extended to $t=32.0$. Simulations were carried out using four groups of single 
males and four groups of single females, resulting in 16 possible pair-types. The initial numbers used for single males, by group, were: $m_{1}=1000, m_{2}=900, m_{3}=800$, and $m_{4}=2700$. For females, the corresponding numbers were: $f_{1}=2000, f_{2}=1000, f_{3}=500$, and $f_{4}=3500$. The initial number of pairs was constrained to zero for all possible pair-types. Removal rates for individuals-whether single or in pairs-were held constant at 0.1. For this exercise, pair dissolution rates were also assumed to be invariant, but with a 5.0 value. Recruitment rates used for (single) males were the following: $m_{1}=$ $100, m_{2}=90, m_{3}=80$, and $m_{4}=270$; the recruitment rates imputed for (single) females were: $f_{1}=$ 200, $f_{2}=100, f_{3}=50$, and $f_{4}=350$. Pair formation rates for males were set at: $m_{1}=3.5, m_{2}=3.0$, $\mathrm{m}_{3}=2.5$, and $\mathrm{m}_{4}=3.5$; and pair formation rates for females were set at: $\mathrm{f}_{1}=2.5, \mathrm{f}_{2}=2.45, \mathrm{f}_{3}=$ 2.3, and $f_{4}=2.586$. As shown in Figure 3, stability in absolute sizes is reached early-at $t<0.5$ (relative distributions, not shown, expose the same trait). This pattern of very rapid stabilization is maintained when averages are computed from simulations based on as few as 30-to-100 realizations. Variance, expressed as standard deviations, for all possible pair-types also stabilizes at $\mathrm{t}<0.5$ (see Figure 4). However, variation for each of the groups of single individuals, male or female, continues to increase until $10<=t<=12$ (Figures 4 and 5). Further simulations will allow us to ascertain whether the pattern observed beyond $t=12$ indeed reflects stability-or only smaller increments in variation. A simulation with 10,000 or more realizations and a time horizon of $50<=t<=100$ could be necessary to accomplish this.

\section{Conclusions.}

In this article we have introduced a flexible framework for the modeling of contact structures in biology. It can be implemented in stochastic and/or deterministic frameworks. We have provided modeling applications in demography-including demographic models for pairs--, epidemiology, inheritance of cultural traits, and food web dynamics. Recently, we have also estimated the contact structure-as modeled by our mixing matrices-of a student population as a function of sexual or dating activity (see Castillo-Chavez et al. 1992; Hsu Schmitz and Castillo-Chavez 1992). In addition, we have worked on methods for estimating the parameters associated with arbitrary mixing structures (see Rubin et al. 1991; Blythe et al. 1992). Furthermore, the representation theory of mixing matrices as a function of the preference structure of a population, as first developed by Busenberg and Castillo-Chavez $(1989,1991)$, has allowed us to begin studying the role of preference in two-sex mixing populations. In fact, we designed and conducted a behavioral survey of a colllege population in order to estimate the preference and mixing structure of two-sex mixing populations.

This research represents our initial efforts in understanding the role of "social" structures in disease dynamics. Most models in the past assumed a fixed social/behavioral structure. The serious study of the transmission dynamics of HIV pointed out the serious limitations of this approach. We have observed a large number of theoretical advances over the last few years (see Castillo-Chavez 1989; Jewell et al. 1992; Anderson and May 1991; and Hethcote and Van Ark 1992). However, we have just begun to understand the effects of changing contact structures in population dynamics. This is what happens when biology meets mathematics!

\section{Acknowledgements}

This research was partially supported by funds from the Dean and the Office of Sponsored Programs of the College of Agriculture and the Mathematics Science Institute at Cornell University, and by NSF grant DEB-9253570 to C. Castillo-Chavez. This work was completed while C. Castillo-Chavez was a visiting member of the Isaac Newton Institute, Cambridge University. This work was significantly improved after discussions held with Simon Levin and the participants of the Wednesday

Tea Seminar at Simon Levin's Lab at Cornell University. This work also benefited from discussions held with Agustin Lobo, Xiaolong Luo and Richard Root at Cornell University.

\section{LITERATURE CITED}

Akre, B.G. and Johnson, D.M. Switching and sigmoid function response curves by damselfly naiads with alternative prey available. J. Animal Ecol. 48:703-720, 1979.

R. M. Anderson, (ed.). (1982). Population dynamics of infectious diseases: theory and applica.ions. Chapman and Hall, London, New York.

Anderson and May (1991). Infectious diseases of humans. Oxford Science Publications. Great Britain. 
Aron, J. and May, R.M. The population dynamics of malaria pp 123-123 in Anderson, R.M. Population dynamics of infectious diseases. Chapman and Hall, London 1982.

Baggs, I., Freedman, H. I. (1990). A mathematical model for the dynamics of interactions between a unilingual and a bilingual population: persitence vs. extinction. J. Math. Sociol, 16: 51-75.

Bailey, N.T.J. (1975). The Mathematical Theory of Infectious diseases and its applications. Griifin, London.

Blythe, S. P., Castillo-Chavez, C., Palmer, J. and Cheng, M. (1991). Towards unified theory of mixing and pair formation. Math. Biosci. 107: 379-405.

Blythe, S. P., Castillo-Chavez, C., and Casella, G. (1992) Empirical methods for the estimation of the mixing probabilities for socially-structured populations from a single survey sample. Mathematical Population Studies. 3(3): 199-225

Boyd, R. and Richerson, P. J. (1985). Culture and the Evolutionary Process. The University of Chicago Press, Chicago.

Brauer, F. Some applications of the theory of Ordinary differential equations to population growth problems. An.Acad.brasil.Cienc. 48:369-385, 1976.

Busenberg, S. and Castillo-Chavez, C. (1989). Interaction, pair formation and force of infection terms in sexually-transmitted diseases. In: Mathematical and statistical approaches to AIDS epidemiology (C. Castillo-Chavez, ed.), pp. 289-300. Lecture Notes in Biomathematics 83, Springer-Verlag, Berlin, Heidelberg, New York, London, Paris, Tokyo, Hong Kong.

Busenberg, S. and Castillo-Chavez, C. (1991). A general solution of the problem of mixing subpopulations, and its application to risk- and age- structured epidemic models for the spread of AIDS. IMA J. of Mathematics Applied in Med. and Biol., 8:1-29

Castillo-Chavez, C. (ed.) (1989). Mathematical and statistical approaches to AIDS epidemiology. Lecture Notes in Biomathematics 83, Springer-Verlag, Berlin, Heidelberg, New York, London, Paris, Tokyo, Hong Kong (405 pages.)

Castillo-Chavez, C. and Busenberg, S. (1991). On the solution of the two-sex mixing problem. In Proceedings of the International Conference on Differential Equations and Applications to Biology and Population Dynamics, S. Busenberg and M. Martelli (eds.), Lecture Notes in Biomathematics 92, 80-98. Berlin, Heidelberg, New York, London, Paris, Tokyo, Hong Kong, Barcelona, Budapest: Springer-Verlag.

Castillo-Chavez, C., Busenberg, S., and Gerow K. (1991). Pair formation in structured populations. In: Differential Equations with Applications in Biology, Physics and Engineering (J. Goldstein, F. Kappel, W. Schappacher, eds.), pp. 47-65. Marcel Dekker, New York

Castillo-Chavez, C., Shyu, S-F., Rubin, G. and Umbauch, D. (1992) On the estimation problem of mixing/pair formation matrices with applications to models for sexually-transmitted diseases. In: AIDS Epidemiology: Methodology Issues (N. P. Jewell, K. Dietz, V. T. Farewell, eds.), 384402. Birkhäuser, Boston-Basel-Berlin.

Cavalli-Sforza, L. L. and Feldman, M.W. (1981). Cultural Transmission and Evolution. Monographs in Population Biology 16. Princeton University Press, Princeton, New Jersey.

Chesson, J.E. Measuring preference in selective predation. Ecology 59:211-215, 1978.

Chesson, J.E. The estimation and analysis of preference and its relationship to foraging models. Ecology 64:1297-1304, 1983.

Cock, M.J.W. The assessment of preference. J. Animal Ecol. 47:805-816, 1978.

Cohen, J.E., Briand, F. and Newman, C.M. Community Food Webs: Data and Theory. Biomathematics 20 Springer-Verlag, New York, 1990.

Crawford, C. M., Schwager, S. J., and Castillo-Chavez, C. (1990). A methodology for asking sensitive questions among college undergraduates. (Biometrics Unit Technical Report BU-1105M).

Dietz, K. (1988a) On the transmission dynamics of HIV. Math. Biosci. 90, 397-414.

Dietz, K. (1988b) Mathematical models for transmission and control of malaria, in Malaria: Principles and Practice of Malariology, Wernsdorfer, W.H. and McGregor, I. (editors) Churchill Livingstone, Edinburg 1988.

Dietz, K. and Hadeler, K.P. (1988) Epidemiological models for sexually transmitted diseases. J. Math. Biol. 26, 1-25.

Dye, C. Epidemiological significance of vector-parasite interactions. Parasitology 101: 409-415, 1990.

Fredrickson, A.G. (1971) A mathematical theory of age structure in sexual populations: Random mating and monogamous marriage models. Math. Biosci. 20, 117-143. 
Fretwell, S.D. Food chain dynamics: the central theory of ecology? Oikos 50:291-301, 1987.

Friend, W.G., Choy, C.T. and Cartwright, E. The effect of nutrient intake on the development and the egg production of Rhodnius prolixus Stahl (Hemiptera: Reduviidae). Can. J. Zool. 43: 891904, 1965.

Gendron, R.P. Models and mechanisms of frequency-dependent predation. American Naturalist 130:603-623, 1987.

Greenwood, J.J.D. The functional basis of frequency-dependent food selection. Biol. J. Linn. Soc. 23:177-199, 1984.

Hadeler, K.P. (1989a) Pair formation in age-structured populations. Acta Applicandae Mathematicae 14, 91-102.

Hadeler, K.P. (1989b) Modeling AIDS in structured populations. 47th Session of the International Statistical Institute, Paris, August/September. Conf. Proc., C1-2.1: 83-99.

Hadeler, K.P. and Nagoma, K. (1990) Homogeneous models for sexually-transmitted diseases. Rocky ountain Journal of Mathematics. 20, 967-986.

Hastings, A. and Powell, T. Chaos in a three-species food chain. Ecology 72:896-903, 1991.

Hess, A.D. and R.O. Hayes Relative potentials of domestic animals for zooprophilaxis against mosquito vectors of encephalitis. Am. J. Trop. Med. Hyg. 19: 327-334, 1970.

Hethcote, H. W. and Yorke, J. A. (1984). Gonorrhea Transmission Dynamics and Control. Lecture Notes in Biomathematics, Vol 56. Springer-Verlag, New York.

Hethcote, H. W. and Van Ark, J. W. (1992). Modeling HIV Transmission and AIDS in the United States. Lecture Notes in Biomathematics, Vol 95. Springer-Verlag, New York.

Hsu Schmitz, S-F. and Castillo-Chavez, C. (1992). Completion of mixing matrices for nonclosed social networks. (BU-1183-M).

Jewell, N. P., Dietz, K., and Farewell, V. T. (1991). AIDS epidemiology: methodological issues. Birkhäuser, Boston, Basel, Berlin.

Kendall, D.G. (1949) Stochastic processes and population growth. Roy. Statist. Soc., Ser. B 2, 230264.

Keyfitz, N. (1949) The mathematics of sex and marriage. Proceedings of the Sixth Berkeley Symposium 0.7 Mathematical Statistics and Probability. Vol. IV: Biology and Health, 89-108.

Kurtz, T. G. (1970). Solutions of ordinary differential equations as limits of pure jump Markov processes. J. Appl. Probab. 7:49-58.

Kurtz, T. G. (1971). Limit theorems for sequences of jump Markov processes approximating differential equations. J. Appl. Probab. 8:344-56.

Leslie, P.H. (1945). On the use of matrices in certain population mathematics. Biometrika, 33:183212.

Levin, S.A. and Segel, L.A. Models of the influence of predation on aspect diversity in prey populations. J. Math. Biol. 14:253-284, 1982.

Lotka, A. J. (1922). The stability of the normal age distribution. Proceedings National Academy of Sciences. 8: 339-345.

Lotka, A. J. (1923). Contributions to the analysis of malaria epidemiology. Amer. J. Hygiene 3. Jan. Sppl.

Lubkin, S., Hsu Schmitz, S-F., Castillo-Chavez, C. (1992). A framework for Modeling Inheritance of Social Traits. (BU-1182-M).

Luo, X. and Castillo-Chavez, C. (1991). Limit behavior of pair-formation models with large dissolution rates. Journal of Mathematical Systems, Estimation, and Control (accepted).

Luo, X., Castillo-Chavez, C. and Cheng, M. (1991). A probabilistic model of pair formation (Biometrics Unit Technical Report BU-1145-M).

Macdonald, G. The epidemiology and control of malaria. Oxford University Press, 1957.

MacKendrick (1926). Applications of mathematics to medical problems. Proceedings Edinburgh Math. Soc., 44:98-190

McFarland, D.D. (1972) Comparison of alternative marriage models. In (Greville, T. N. E., ed.), Population Dynamics. Academic Press, New York London: 89-106.

Maddox, G.D. and Root, R. Structure of the encounter between goldenrod Solidago altissima and its diverse insect fauna. Ecology 71:2115-2124, 1990.

Molineaux, L. The epidemiology of human malaria as an explanation of its distribution, including some implications for its control pr 913-998 in Malaria: Principles and Practice of Malariology, Wernsdorfer, W.H. and McGregor, 1. (editors) Churchill Livingstone, Edinburg 1988.

Molineaux, L., and Gramiccia, G. The gerki project. World Health Organization, Geneva 1980. 
Molineaux, L., Muir, D.A., Spencer, H.C. and Wernsdorfer, W.H. The epidemiology of malaria and its measurement pp. 999-1090 in Malaria: Principles and Practice of Malariology, Wernsdorfer, W.H. and McGregor, I. (editors) Churchill Livingstone, Edinburg 1988.

Molyneaux, D.H. and Jefferies, D. Feeding behaviour of pathogen-infected vectors. Parasitology 92: 721-736, 1986.

Nold, A. (1980) Heterogeneity in disease-transmission modeling. Math. Biosci. 52, 227-240.

Oaten, A. and Murdoch, W.W. Switching, functional response, and stability in predator prey systems. Amer. Natural. 109:299-318, 1975.

Pacala, S.W. and Dobson, A.P. The relation between the number of parasites/host and host age: population dynamic causes and maximum likelihood estimation. Parasitology 96: 197-210.

Paine, R.T. Food-web analysis through field measurement of per capita interaction strength. Nature 355: 73-75, 1992.

Parlett, B. (1972) Can there be a marriage function? In (Greville, T. N. E., ed.), Population Dynamics. Academic Press, New York London: 107-135.

Pimm, S.L. Food Webs. Chapman and Hall, London, 1982.

Pimm, S.L. The geometry of niches. In Community Ecology (A. Hastings, editor). Lecture Notes in Biomathematics 77, Springer-Verlag, New York, 1988.

Pimm, S.L. and Kitching, R.L. Food web patterns: trivial flaws or the basis of an active research program? Ecology 69:1669-1672, 1988.

Polis, G. Complex trophic interactions in deserts: an empirical critique of food web theory. Amer. Natural. 138:123-155, 1991.

Pollard, J.H. (1973) Mathematical models for the growth of human populations, Chapter 7: The two sex problem. Cambridge University Press.

Price, P.W. Insect Ecology. John Wiley, New York, 1984.

Ribeiro, J.M., Rosignol, P.A. and Spielman, A. Aedes aegypti: model for blood finding strategy and prediction of parasite manipulation. Exp. Parasitol. 60: 118-132, 1985.

Rogers, D.J. A general model for the African trypanosomiasis. parasitology 97: 193-212, 1988.

Ross, R. The prevention of malaria (2nd edition, with addendum) John Murray, London, 1911.

Rubin, G., Umbauch, D., Shyu, S-F., and Castillo-Chavez, C. (1992) Application of capturerecapture methodology to estimation of size of population at risk of AIDS and/or other sexuallytransmitted diseases. Statistics in Medicine. 11: 1533-1549

Schoefield, C.H. The role of blood intake in density regulation of populations of Triatoma infestans (Klug)(Homoptera: Reduviidae). Bul. Ent. Res 72: 617-629, 1982.

Schwager, S., Castillo-Chavez, C., and Hethcote, H. (1989). Statistical and mathematical approaches to AIDS epidemiology: A review. In: Mathematical and statistical approaches to AIDS epidemiology (C. Castillo-Chavez, ed.), pp. 2-35. Lecture Notes in Biomathematics 83, SpringerVerlag, Berlin, Heidelberg, New York, London, Paris, Tokyo, Hong Kong.

Teramoto, E., Kawasaki, K. and Shigesada, N. Switching effect of predation on competitive prey species. J. Theor. Biol. 79:303-315, 1979.

Tilman, D. (1982). Resource competition and community structure. Princeton University Press, Princeton, N. J.

Velasco-Hernández, J. X. and Castillo-Chavez, C. (1991) Food web structure and frequency dependent predation. (Biometrics Unit Technical Report BU-1142-M).

Velasco-Hernández, J. X. (1991). An epidemiological model for the dynamics of Chagas' disease. Biosystems, 26:127-134.

Waldstätter, R. (1989) Pair formation in sexually transmitted diseases. In: Mathematical and statistical approaches to AIDS epidemiology (C. Castillo-Chavez, ed.), pp. 260-274. Lecture Notes in Biomathematics 83, Springer-Verlag, Berlin, Heidelberg, New York, London, Paris, Tokyo, Hong Kong.

Zeledon, R. and Rabinovich, J.E. Chagas' disease: an ecological appraisal with special emphasis on its insect vector. Ann. Rev. Entomol. 26: 101-103, 1981. 
Figure 1. Simulation Results for the Food Web Model, with $b=4$ and $e=80$. The Simulation Was Run for 5,000 Time Units.

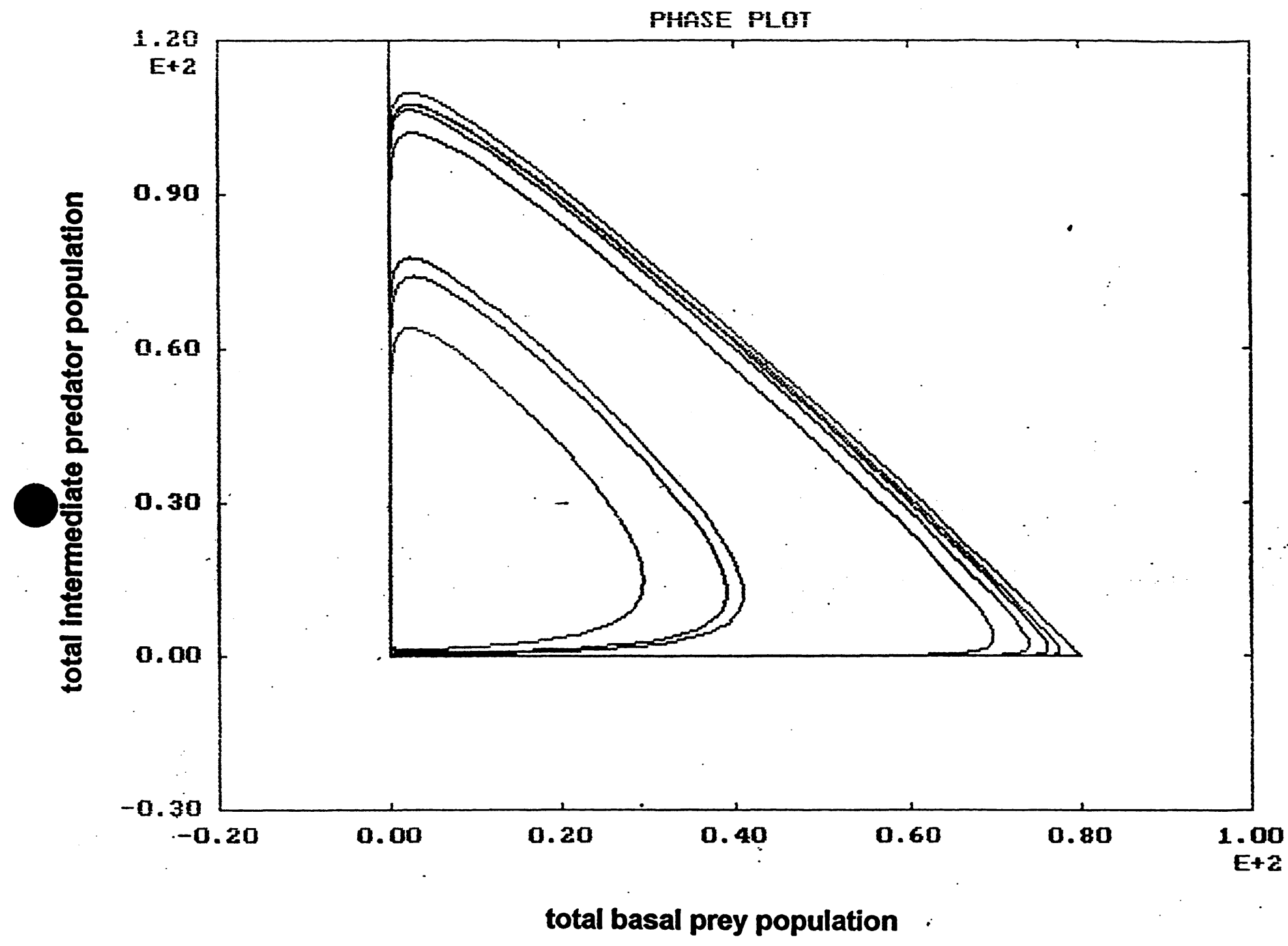


Figure 2. Simulation Results for the Food Web Model, with $b=4$, and $e=2$.

The Plot Includes Only Results Between 20,000 and 30,100 Time Units.

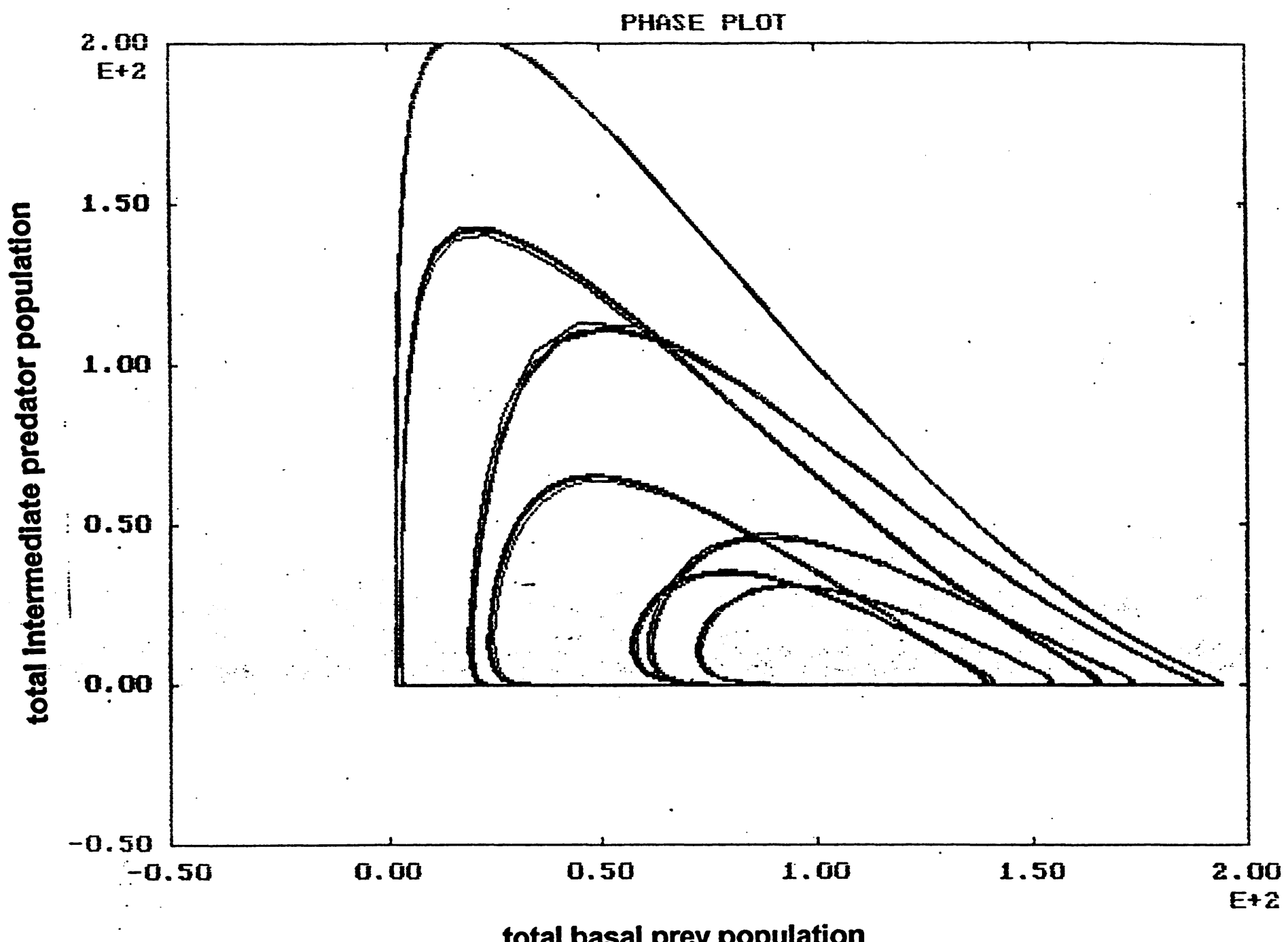


Figure 3. Total Population by Sex, Class, and Pairing Groups.

Simulation with $\sigma=5.0, t<=2.0$, and 10,000 Realizations.

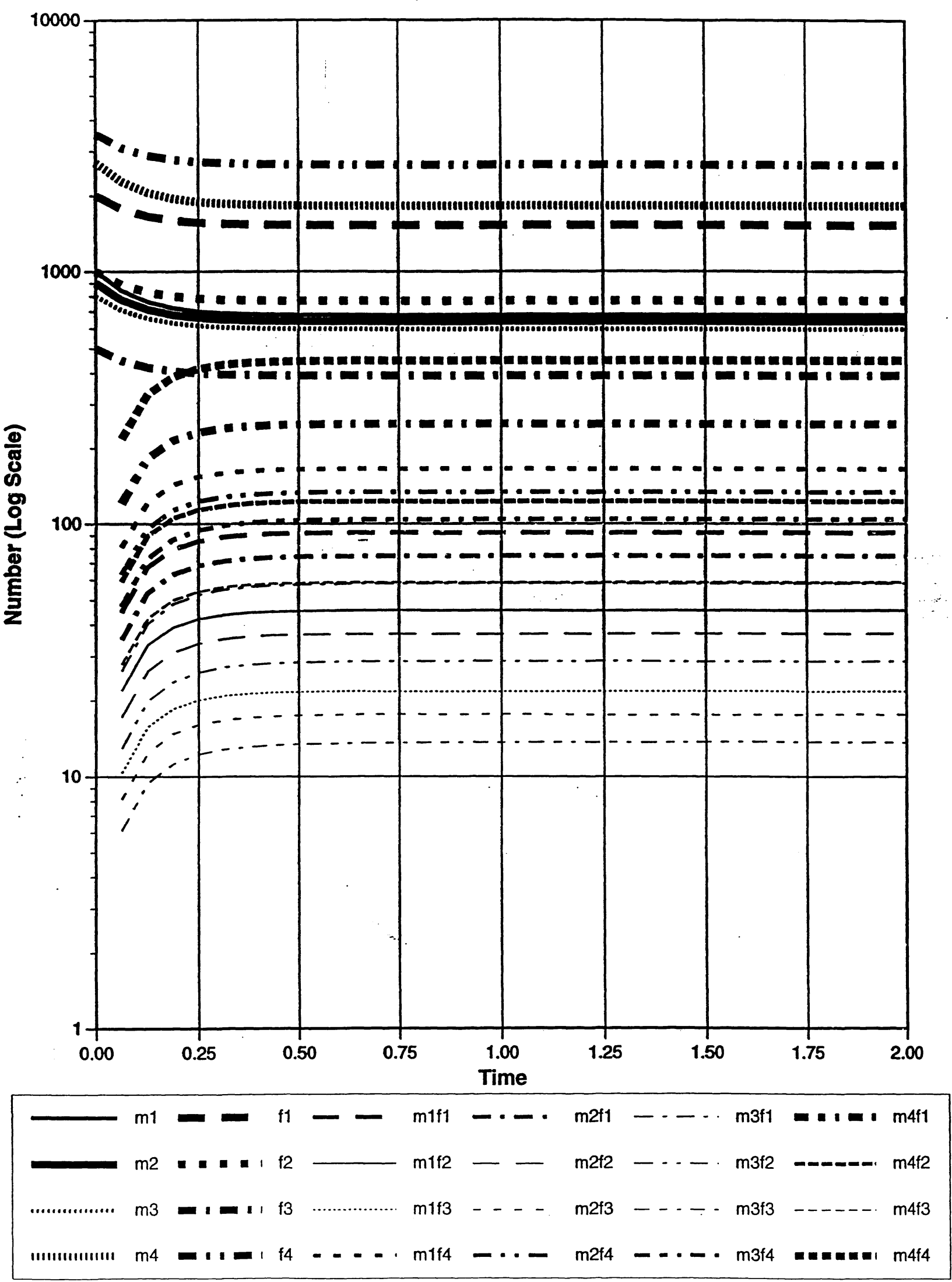


Figure 4. Standard Deviation, Total Population by Sex, Class, and Pairing Groups. Simulation with $\sigma=5.0, t<=32.0$, and 10,000 Realizations.

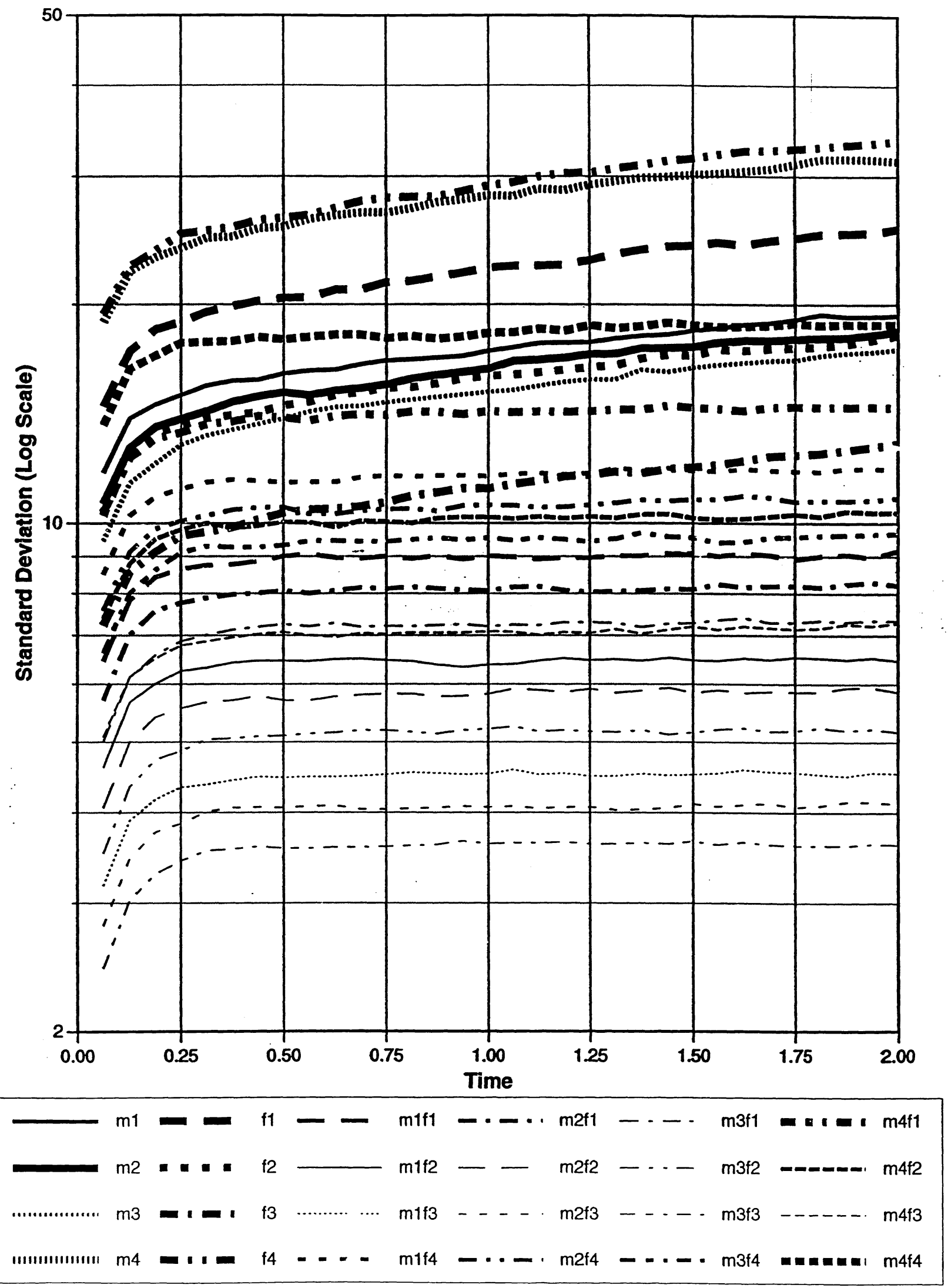


Figure 5. Standard Deviation, Total Population by Sex, Class, and Pairing Groups. Simulation with $\sigma=5.0, t<=32.0$, and 1,000 Realizations.

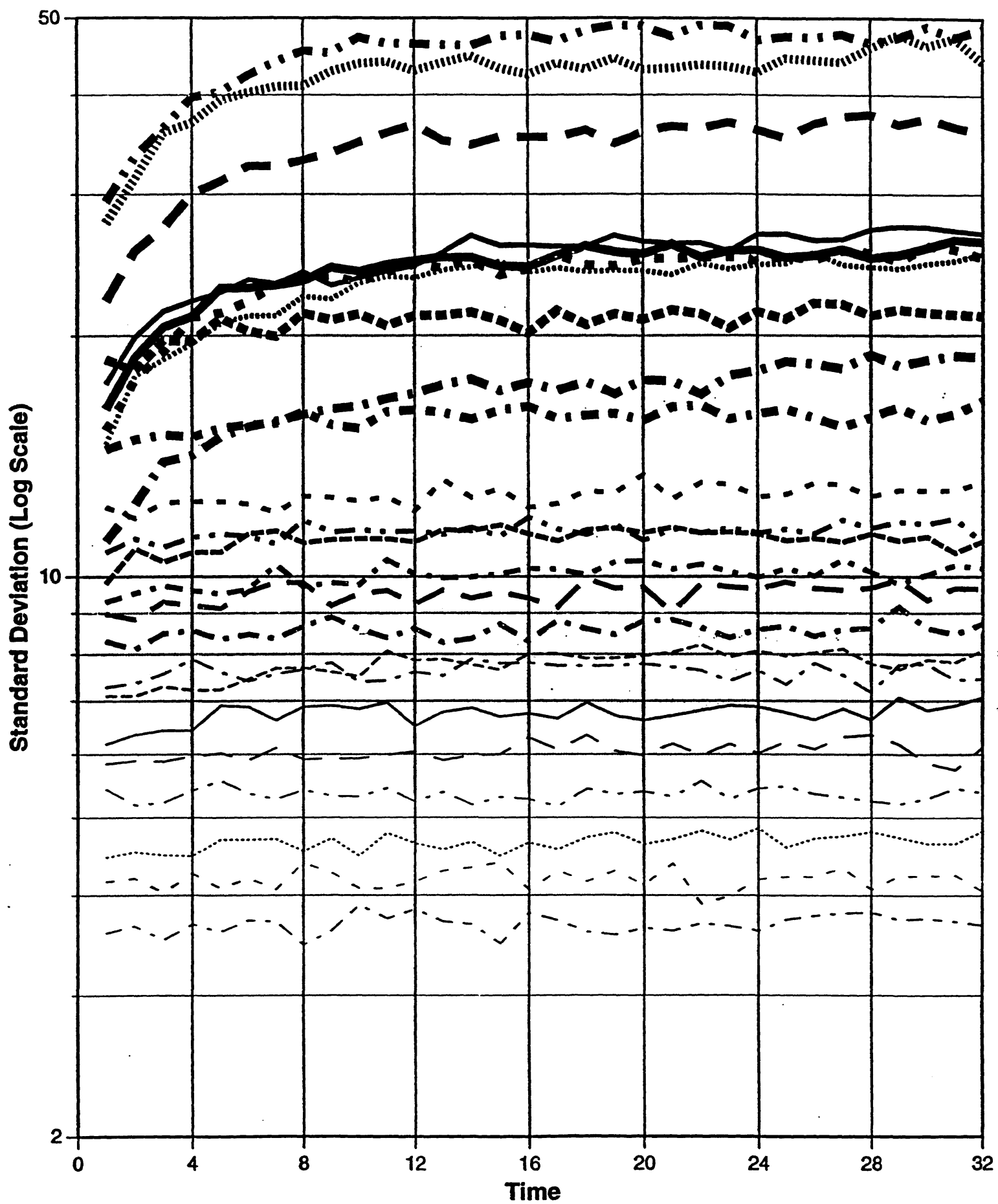

\begin{tabular}{|c|c|c|c|c|c|c|c|c|c|c|}
\hline & $\mathrm{m} 1$ & $=$ & $f 1$ & - & $\mathrm{m} 1 \mathrm{f1}$ & $-\cdots$ & $m 2 f 1$ & -- & $m 3 f 1$ & $=\because=m 4 \mathrm{ft}$ \\
\hline & $\mathrm{m} 2$ & 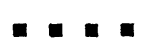 & †2 & - & $m 1 f 2$ & -- & $\mathrm{m} 2 \mathfrak{2} 2$ & $\cdots-$ & $m 3 \mathfrak{m} 2$ & - - - \\
\hline ……............. & $\mathrm{m} 3$ & $=1=1$ & $\uparrow 3$ & .................... & 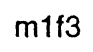 & $-\ldots$ & $m 2 \nmid 3$ & $\cdots \cdots$ & 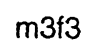 & $\ldots \ldots m 4 f 3$ \\
\hline 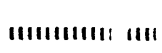 & m4 & $=1.1$ & $\mathrm{f} 4$ & $=-$ & $m 1 f 4$ & $-\cdots-$ & 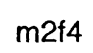 & $-\cdots-$ & $m 3 f 4$ & $m=-m=m \quad m 4 f 4$ \\
\hline
\end{tabular}

